TRANSACTIONS OF THE

AMERICAN MATHEMATICAL SOCIETY

Volume 357, Number 7 , Pages 2571-2611

S 0002-9947(05)03880-8

Article electronically published on March 1, 2005

\title{
ON NONLINEAR WAVE EQUATIONS WITH DEGENERATE DAMPING AND SOURCE TERMS
}

\author{
VIOREL BARBU, IRENA LASIECKA, AND MOHAMMAD A. RAMMAHA
}

\begin{abstract}
In this article we focus on the global well-posedness of the differential equation $u_{t t}-\Delta u+|u|^{k} \partial j\left(u_{t}\right)=|u|^{p-1} u$ in $\Omega \times(0, T)$, where $\partial j$ is a sub-differential of a continuous convex function $j$. Under some conditions on $j$ and the parameters in the equations, we obtain several results on the existence of global solutions, uniqueness, nonexistence and propagation of regularity. Under nominal assumptions on the parameters we establish the existence of global generalized solutions. With further restrictions on the parameters we prove the existence and uniqueness of a global weak solution. In addition, we obtain a result on the nonexistence of global weak solutions to the equation whenever the exponent $p$ is greater than the critical value $k+m$, and the initial energy is negative. We also address the issue of propagation of regularity. Specifically, under some restriction on the parameters, we prove that solutions that correspond to any regular initial data such that $u_{0} \in H^{2}(\Omega) \cap H_{0}^{1}(\Omega)$, $u_{1} \in H_{0}^{1}(\Omega)$ are indeed strong solutions.
\end{abstract}

\section{INTRODUCTION}

1.1. The model. Let $j(s)$ be a continuous, convex real-valued function defined on $\mathbb{R}$ and let $\partial j$ be its sub-differential [3. Let $\Omega$ be a bounded domain in $\mathbb{R}^{n}$ with a smooth boundary $\Gamma$.

This paper is concerned with the solvability of the following initial-boundary value problem:

$$
\begin{gathered}
u_{t t}-\Delta u+|u|^{k} \partial j\left(u_{t}\right)=|u|^{p-1} u \quad \text { in } \Omega \times(0, T) \equiv Q_{T}, \\
u(x, 0)=u_{0}(x) \in H_{0}^{1}(\Omega), \quad u_{t}(x, 0)=u_{1}(x) \in L_{2}(\Omega), \\
u=0 \text { on } \Gamma \times(0, T),
\end{gathered}
$$

where the problem is studied under the following condition imposed on the convex function $j$ and the parameters $k, m, p$.

Assumption 1.1. $k, m, p \geq 0$. In addition, $k \leq \frac{n}{n-2}, p+1<\frac{2 n}{n-2}$, if $n \geq 3$.

- Coercivity condition: $j(s) \geq c|s|^{m+1}$, where $c>0$.

- Strict monotonicity: $(\partial j(s)-\partial j(v))(s-v) \geq c_{1}|s-v|^{m+1}$, where $c_{1}>0$.

- Continuity: $\partial j(s)$ is single valued and $|\partial j(s)| \leq c_{0}|s|^{m}+c_{2}$, for some constants $c_{0}>0, c_{2} \geq 0$.

Received by the editors March 31, 2003.

2000 Mathematics Subject Classification. Primary 35L05, 35L20; Secondary 58J45.

Key words and phrases. Wave equations, damping and source terms, generalized solutions, weak solutions, blow-up of solutions, sub-differentials, energy estimates. 
In this paper, we study the long-time behavior of solutions to the initial-boundary value problem (1.1). Of central interest is the relationship of the source and damping terms to the behavior of solutions.

Interestingly, the partial differential equation in (1.1) is a special case of the prototype evolution equation

$$
u_{t t}-\Delta u+\mathcal{R}\left(x, t, u, u_{t}\right)=\mathcal{F}(x, u),
$$

where in (1.2) the nonlinearities satisfy the structural conditions $v \mathcal{R}(x, t, u, v) \geq 0$, $\mathcal{R}(x, t, u, 0)=\mathcal{F}(x, 0)=0$, and $\mathcal{F}(x, u) \sim|u|^{p-1} u$ for large $|u|$. Various special cases of (1.2) arise in quantum field theory and some important mechanical applications. See for example Jörgens [11] and Segal [26].

A benchmark equation, which is a special case of (1.1), is the following wellknown polynomially-damped wave equation studied extensively in the literature (see for instance [22, 24]):

$$
\begin{gathered}
u_{t t}-\Delta u+|u|^{k}\left|u_{t}\right|^{m-1} u_{t}=|u|^{p-1} u \quad \text { in } \Omega \times(0, T), \\
u(x, 0)=u^{0}(x) \quad u_{t}(x, 0)=u^{1}(x) \quad \text { in } \Omega, \\
u(x, t)=0 \text { on } \Gamma \times(0, T) .
\end{gathered}
$$

Indeed, by taking $j(s)=\frac{1}{m+1}|s|^{m+1}$, then obviously $\partial j(s)=|s|^{m-1} s$, and therefore Assumption 1.1 is satisfied. It is also easy to see in this case that problem (1.1) is equivalent to (1.3).

It is worth noting here that when the damping term $|u|^{k}\left|u_{t}\right|^{m-1} u_{t}$ is absent, the source term $|u|^{p-1} u$ drives the solution of (1.3) to blow up in finite time [7, 15. 21, 32. In addition, if the source term $|u|^{p-1} u$ is removed from the equation, then damping terms of various forms are known to yield existence of global solutions (cf. [2, 3, 4, 10]). However, when both damping and source terms are present in the equation, then the analysis of their interaction and their influence on the global behavior of solutions becomes more difficult. We refer the reader to [6, 16, 18, 22, 24, 27, 30] and the references therein.

It should be noted that if $k=0$ and $p=0$, then equation (1.1) can be treated via the theory of monotone operators and the full well-posedness of strong solutions (in the terminology of monotone operator theory) is now classical [3]. In addition, with $k=0$, the presence of a locally Lipschitz source term from $H^{1}(\Omega)$ into $L_{2}(\Omega)$ does not affect the arguments for establishing the existence of local solutions via perturbation theory of monotone operators. Moreover, if $p \leq k+m$, then one can derive the necessary a priori bounds that guarantee that every local solution is indeed global in time.

The situation is however different when the damping term is degenerate, leading to the degeneracy of the monotonicity argument (problems of this type often arise in physics when the friction is modulated by the strains). In fact, when $k>0,(1.3)$ is no longer a locally Lipschitz perturbation of a monotone problem (even in the case when $p \leq \frac{n}{n-2}$, i.e., the source term is a locally Lipschitz function from $H^{1}(\Omega)$ into $L_{2}(\Omega)$ ). Thus, standard monotone operator theory and the celebrated method of Lions and Strauss [20] do not apply. This fact, combined with a potential strong growth of the damping term (the case when $m>1$ ), makes the problem interesting and the analysis more subtle. In fact, one needs to be careful about the meaning of the solution and its relation to the equation. 
The goal of our paper is to provide results on the existence and uniqueness of various types of solutions such as generalized solutions, weak solutions and strong solutions.

1.2. Main results. In order to proceed with the presentation of our results we shall introduce the appropriate definitions. First, we give the definition of a generalized solution, which satisfy a certain variational inequality. In discussing finite energy solutions (i.e., $\left.\left(u, u_{t}\right) \in H^{1}(\Omega) \times L_{2}(\Omega)\right)$ we shall impose another restriction on the parameters $p, m, k$

$$
p \leq \max \left\{\frac{p^{*}}{2}, \frac{p^{*} m+k}{m+1}\right\} ; p^{*} \equiv \frac{2 n}{n-2} .
$$

Remark 1.2. We note here that the range of values of the parameter $p$ is beyond what is required for the source term to be a locally Lipschitz function from $H^{1}(\Omega)$ into $L_{2}(\Omega)$ ), as typically assumed in the literature. For instance, in [6] the restriction $p<\frac{p^{*}}{2}$ was crucial, in the nondegenerate case $k=0$, for establishing the existence of local and global solutions of finite energy on a bounded domain. Instead, condition (1.4) allows "supercritical" values of $p$ provided $p^{*}(m-1)+2 k>0$. Analogous conditions in 27] are imposed in the nondegenerate case $k=0$, where finite energy solutions with compactly supported data in $\mathbb{R}^{n}$ are constructed. We also note that condition (1.4) turns out to be essential only when one is dealing with finite energy solutions in $H^{1}(\Omega) \times L_{2}(\Omega)$. However, in the case of higher regularity solutions, discussed later in Theorem 1.10, condition (1.4) will be relaxed even further.

Definition 1.3. A function $u \in C_{w}\left([0, T], H_{0}^{1}(\Omega)\right) \cap C_{w}^{1}\left([0, T], L_{2}(\Omega)\right)$ with $|u|^{k} j\left(u_{t}\right)$ $\in L_{1}(\Omega \times(0, T))$ and under the condition (1.4) is said to be a generalized solution to (1.1) if and only if for all $0<t \leq T$ the following inequality holds:

$$
\begin{array}{r}
\int_{0}^{t} \int_{\Omega}\left(u_{t} v_{t}-\nabla u \nabla v\right) d x d t+1 / 2 \int_{\Omega}\left[u_{t}^{2}(t)+|\nabla u(t)|^{2}\right] d x \\
+\int_{0}^{t} \int_{\Omega}|u|^{k}\left[j\left(u_{t}\right)-j(v)\right] d x d t \\
\leq \int_{0}^{t} \int_{\Omega}|u|^{p-1} u\left(u_{t}-v\right) d x d t+1 / 2 \int_{\Omega}\left[u_{1}^{2}+\left|\nabla u_{0}\right|^{2}-2 u_{1} v(0)\right] d x
\end{array}
$$

for all test functions $v$ satisfying

$$
v \in H^{1}\left(0, T ; L_{2}(\Omega)\right) \cap L_{2}\left(0, T ; H_{0}^{1}(\Omega)\right) \cap L_{\infty}(\Omega \times(0, T)), v(t)=0 .
$$

Remark 1.4. We note here that, if $u$ is a generalized solution to (1.1), then $u$ satisfies $|u|^{k} \partial j\left(u_{t}\right) \in L_{r}\left(Q_{t}\right)$, where $r=\frac{p^{*}(m+1)}{k+p^{*} m}>1$. Moreover $|u|^{p}\left|u_{t}\right| \in L_{1}\left(Q_{t}\right)$. The last assertion follows from condition (1.4). Indeed, if $p \leq \frac{p^{*}}{2}$, then the Cauchy-Schwartz inequality and the a priori $H^{1}(\Omega) \times L_{2}(\Omega)$ regularity of solutions imply the desired conclusion. If, instead, $\frac{p^{*}}{2}<p \leq \frac{p^{*} m+k}{m+1}$, then we must have $p(m+1)-k>0$. Consequently, by Hölder's and Young's inequalities we have

$$
\begin{aligned}
\int_{Q_{t} \cap\{|u| \geq 1\}}|u|^{p}\left|u_{t}\right| d x d t & \leq \epsilon \int_{Q_{t}}|u|^{k}\left|u_{t}\right|^{m+1} d x d t+C_{\epsilon} \int_{Q_{t} \cap\{|u| \geq 1\}}|u|^{\frac{p(m+1)-k}{m}} d x d t \\
& \leq \epsilon \int_{Q_{t}}|u|^{k} j\left(u_{t}\right) d x d t+C_{\epsilon} \int_{0}^{t}|u|_{H^{1}(\Omega)}^{\frac{p(m+1)-k}{m}} d t
\end{aligned}
$$


where we have used condition (1.4) along with the Sobolev embedding theorem and the coercivity condition in Assumption 1.1. and where $\epsilon>0$ is suitably small. Thus, all the terms in the definition of generalized solution are well defined for the class of solutions and the considered parameters.

It should be noted here that Definition 1.3 is a proper extension of the notion of classical solutions. Indeed, if $u$ is a sufficiently smooth generalized solution, then $u$ satisfies the classical definition of "weak" solution. To see this it suffices to take in Definition 1.3 the test function $v(t)=u_{t}(t)+\psi(t)$, where $\psi \in H^{1,1}\left(Q_{t}\right) \cap L_{\infty}\left(Q_{t}\right)$. Integration by parts and accounting for cancellation of terms yields

$\int_{Q_{t}}\left(\Delta u-u_{t t}\right) \psi d Q_{t}+\int_{0}^{t} \int_{\Omega}|u|^{k}\left(j\left(u_{t}\right)-j\left(u_{t}+\psi\right)\right) d x d t+\int_{0}^{t} \int_{\Omega}|u|^{p-1} u \psi d x d t \leq 0$,

where the above relation can be extended by density to all $\psi \in L_{2}\left(Q_{T}\right)$. By taking $\psi \equiv \lambda \phi, \lambda>0, \phi \in L_{2}\left(Q_{t}\right)$, and then by letting $\lambda \rightarrow 0$, we obtain

$$
\int_{Q_{t}}\left(\Delta u-u_{t t}\right) \phi d Q_{t}-\int_{0}^{t} \int_{\Omega}|u|^{k} \partial j\left(u_{t}\right) \phi d x d t+\int_{0}^{t} \int_{\Omega}|u|^{p-1} u \phi d x d t \leq 0 .
$$

Due to arbitrariness of $\phi$, the inequality above can be replaced by equality, as claimed.

Our second (more stringent) definition of solution is given as follows.

Definition 1.5. A function $u \in C\left([0, T], H_{0}^{1}(\Omega)\right) \cap C_{w}^{1}\left([0, T], L_{2}(\Omega)\right)$ which satisfies $\Delta u-u_{t t}$ and $|u|^{k} j\left(u_{t}\right) \in L_{2}(\Omega \times(0, T))$ is said to be a weak solution to (1.1) if and only if, for all $0<t \leq T$, the following variational equality holds:

$$
\begin{array}{r}
\int_{0}^{t} \int_{\Omega}\left(u_{t t}-\Delta u\right) v d x d t+\int_{0}^{t} \int_{\Omega}|u|^{k} \partial j\left(u_{t}\right) v d x d t \\
=\int_{0}^{t} \int_{\Omega}|u|^{p-1} u v d x d t \\
u(0)=u_{0}, u_{t}(0)=u_{1}
\end{array}
$$

for all test functions $v \in L_{2}(\Omega \times(0, T))$.

Equivalently, we may write

$$
\begin{array}{r}
\int_{0}^{t} \int_{\Omega}\left(-u_{t} v_{t}+\nabla u \nabla v\right) d x d t-\int_{\Omega} u_{1} v(0) d x+ \\
\int_{0}^{t} \int_{\Omega}|u|^{k} \partial j\left(u_{t}\right) v d x d t \\
=\int_{0}^{t} \int_{\Omega}|u|^{p-1} u v d x d t
\end{array}
$$

for all test functions $v$ satisfying

$$
v \in H^{1}\left(0, T ; L_{2}(\Omega)\right) \cap L_{2}\left(0, T ; H_{0}^{1}(\Omega)\right), v(t)=0 .
$$

It should be noted here that Definition 1.5 is equivalent to

$$
\square u=-|u|^{k} \partial j\left(u_{t}\right)+|u|^{p-1} u, \text { a.e. }(x, t) \in \Omega \times(0, T),
$$

where $\square \equiv \frac{d^{2}}{d t^{2}}-\Delta$ is understood in the sense of distributions.

At this end, we remark that the following notation will be used in the sequel:

$$
|u|_{s, \Omega} \equiv|u|_{H^{s}(\Omega)} \text { and }\|u\|_{p} \equiv\|u\|_{L_{p}(\Omega)},
$$


where $H^{s}(\Omega)$ and $L_{p}(\Omega)$ stand for the classical Sobolev spaces and the Lebesgue spaces, respectively. Also, we let $A: L^{2}(\Omega) \rightarrow L^{2}(\Omega)$, where $A=-\Delta$ with its domain $\mathcal{D}(A)=H^{2}(\Omega) \cap H_{0}^{1}(\Omega)$.

Our main result, which establishes local and global existence of generalized solutions, reads as follows:

Theorem 1.6 (Generalized solutions). Under Assumption 1.1 and condition (1.4), there exists a local generalized solution to (1.1) defined on $\left(0, T_{0}\right)$ for some $T_{0}>0$. If, in addition, $p \leq k+m$, then the said generalized solution is global and $T_{0}$ may be taken arbitrarily large.

Remark 1.7. If $k=0$, then the variational inequality in (1.5) becomes equality and the solution $u$ is unique and satisfies the equation in the sense of (1.8) with $j\left(u_{t}\right) \in L_{1}\left(Q_{t}\right), u_{t} \in L_{m+1}\left(Q_{t}\right)$. It should be pointed out that the main difficulty of the problem under consideration in Theorem [1.6] is the fact that the damping term is not monotone and degenerate $(k>0)$. This difficulty goes away when $k=0$. As shown later (see Remark [3.7), the proof of Theorem 1.6 simplifies drastically when $k=0$ and our arguments lead to stronger conclusions. In particular, the strong monotonicity allows us to replace inequalities by equalities. Thus, for $k=0$ one obtains the existence theory which is consistent with the literature and provides an extension to a larger "supercritical" set for the parameter $p$, namely, $p \geq \frac{n}{n-2}$ (see [6] for details).

Having obtained generalized solutions (with no restrictions on parameter $m$ ) one would wonder how these solutions relate to weak solutions that have been obtained in the literature 22] but only in the special case when $k>0, j(s)=\frac{1}{m+1}|s|^{m+1}$, $m<1$, and subject to (1.9). In fact, as pointed out below, for these special cases with additional restrictions imposed on the parameters $k, m$, our generalized solutions become unique weak solutions in the sense of Definition 1.5 Thus a particular specialization of Theorem 1.6 to a much narrower range of parameters fully recovers and generalizes (to a larger class of damping functions $j(s)$ ) the recent results of [22]. Indeed, a particular specialization of Theorem 1.6 yields the following corollary.

Corollary 1.8. In addition to Assumption 1.1 and condition (1.4) we also assume that

$$
\left\{\begin{array}{l}
m<1 \text { if } n=1,2 \\
\frac{k}{p^{*}}+\frac{m}{2} \leq \frac{1}{2} \text { if } n \geq 3 .
\end{array}\right.
$$

Then, there exists a local weak solution to 1.1) which is defined on an interval $(0, T)$, for some $T>0$. Moreover, we have:

- If $p \leq k+m$, then the said solution is global and $T$ may be taken arbitrarily large.

- If $k, p \geq 1$, then the said solution is unique and depends continuously on the initial data.

An interesting question that one should ask next is what happens when $m=1$ ? We do have generalized solutions, as asserted by Theorem[1.6. But do we have weak solutions? If so, are these solutions unique? A positive answer to this question is provided by the next theorem. 
Theorem 1.9 (The case $m=1$ ). In addition to Assumption 1.1, assume that $k+1 \leq \frac{p^{*}}{2}$. Then, there exists a local weak solution $u$ to (1.3) (i.e., when $j(s)=$ $\frac{1}{2}|s|^{2}$ and $\left.\partial j(s)=s\right)$ such that $u \in C_{w}\left([0, T], H_{0}^{1}(\Omega)\right) \cap C_{w}\left([0, T], L_{2}(\Omega)\right), \Delta u-u_{t t} \in$ $L_{1}(\Omega \times(0, T))$, where $u$ satisfies (1.7) with the test functions $v \in L_{\infty}(\Omega \times(0, T))$, and $T$ may be finite. In addition, we have:

- If $p \leq k+1$, then the said weak solution is global and $T$ may be taken arbitrarily large.

- If $p \geq 1$, then such a solution $u$ is unique, but it may not be continuously dependent on the initial data in the finite energy norm.

Our next theorem addresses the issue of propagation of regularity. This means that more regular data produce more regular solutions. In fact, the result below states that this is always the case locally (i.e., for sufficiently small times). However, in the special case when the parameter $p$ is below the critical value $k+m$, then the propagation of regularity is a global phenomena.

Theorem 1.10 (Strong (regular) solutions). With the validity of Assumption 1.1, further assume that $n<5$ and

$$
k \geq 1,2 \leq p<\frac{4}{n-2}+1, m+1<\frac{n}{n-2}, k+m<\frac{4}{n-2}+1 .
$$

Then, for every initial data satisfying $u_{0} \in H^{2}(\Omega) \cap H_{0}^{1}(\Omega), u_{1} \in H_{0}^{1}(\Omega)$, there exists $T_{0}>0$ such that (1.1) has a unique local solution $u$ with the regularity that $u \in C\left([0, T], H^{2}(\Omega)\right) \cap C^{1}\left([0, T], H^{1}(\Omega)\right)$, for some $T \leq T_{0}$ where $T_{0}$ may be finite.

In addition, if we assume that $p \leq k+m, p \leq \frac{p^{*}}{2}$, and either $k=0$ or else $\frac{k}{p^{*}}+\frac{m}{2} \leq \frac{1}{2}$, then regular solutions are global and $T_{0}$ can be taken arbitrarily large.

Remark 1.11. We note here that the second part of Theorem 1.10 provides regular solutions in the context of Corollary 1.8. Thus, the weak solutions established in Corollary 1.8 become regular provided the initial data are taken in $H^{2}(\Omega) \cap H_{0}^{1}(\Omega) \times$ $H^{1}(\Omega)$.

Remark 1.12. For $n<4$ our proof of Theorem 1.10 allows us to assume that $m+1 \leq \frac{n}{n-2}$ and $k+m \leq \frac{4}{n-2}+1$ instead of the strict inequalities as assumed in the statement of Theorem 1.10 .

Finally, we shall address the issue of a strong source (large values of $p$ when $p>k+m$ ) which may lead to a finite-time blow up of solutions. Here, our results are inspired by [6], where the question of finite time blow up in the presence of damping in wave equations has been addressed first and solved optimally. The arguments of [6] were later generalized to a larger class of damped hyperbolic like dynamics 16] and more recently adapted in [22] in order to treat blow up of solutions in the degenerate case with $k>0, j(s)=|s|^{m+1}$ and subject to condition (1.9). We also wish to point out that the blow-up region $p>k+m$ for the degenerate case was identified for the first time in [16]. The blow-up techniques developed in [6] and their generalization in 16, 22, are applicable to weak solutions as given in Definition 1.5. In fact, the core of the proof, including the construction of an appropriate "Liapunov's" function, is essentially the same in these works. However, for sake of completeness we briefly include this aspect of the analysis as well. 
Theorem 1.13. Assume the validity of Assumption 1.1 with $c_{2}=0$ and that $p>k+m$. In addition, assume that $E(0)<0$, where $E(0)$ is the initial energy given by

$$
E(0)=\frac{1}{2}\left(\left|u_{1}\right|_{0, \Omega}^{2}+\left|A^{1 / 2} u_{0}\right|_{0, \Omega}^{2}\right)-\frac{1}{p+1}\left\|u_{0}\right\|_{L^{p+1}(\Omega)}^{p+1} .
$$

Then, the weak solution to (1.1) blows up in a finite time.

\subsection{Comments about our results and their relationship to the literature.}

- Our first new result stated in Theorem 1.6 provides local and global existence result in the challenging case when $m>1$. It also includes previous results established in 6, 22, 24] and other references. As we have already mentioned above, the particular feature of the problem studied in this paper is the potential degeneracy of the damping term. Thus, the beneficial effects of damping may not be present when the displacement have strong oscillations. In fact, the special case (1.3) was studied first in [22] with sub-linear damping in the velocity, i.e., $m<1$ and subject to $p, k \geq 1$ and $\frac{k}{p^{*}}+\frac{m}{2} \leq \frac{1}{2}$. In this case and with the assumption that $p \leq k+m$, the authors in 22] established the existence of a unique global weak solution. When $m>1$, the situation is more complex. This is mostly due to the notorious difficulties in passing to the limit in a super-linear term in $u_{t}$ with a weakly convergent subsequence. The lack of good structure, namely the lack of monotonicity, and super-linearity of $u_{t}$ in a degenerate damping term render standard tools nonapplicable. Nevertheless, our result in Theorem 1.6 establishes the existence of a global generalized solution with no restrictions on $m>0$. In addition, the range of the parameter $p$ allowed in Theorem 1.6 is larger than what is typically assumed in the literature, even when $k=0$.

- In view of the existence and uniqueness of weak solutions to (1.3) in the case $m<1$ and under assumption (1.9) (see [22]), it was natural to address the question of how our generalized solutions obtained in Theorem 1.6 relate to the ones obtained in 22]. A positive answer to this question is given in Corollary 1.8, where we show that our generalized solutions obtained in Theorem 1.6] are indeed the same solutions obtained in [22], provided the parameters are restricted to a narrower range of values. Corollary 1.8 not only establishes the equivalence of these two classes of solutions for a restrictive set of parameters (whenever they are comparable), but it also generalizes the result of 22 to a larger class of damping terms described by the sub-differential $\partial j(s)$.

- The case $m=1$ and $k>0$ treated in Theorem 1.9 is, to the best of our knowledge, new in the literature. Here, the main issue is to prove uniqueness of solutions. This is accomplished by using a certain change of variables which reduces treatment of the problem to that of a parabolic equation.

- Theorem[1.10 deals with more regular solutions which are unique and satisfy the classical variational definition of solutions. To the best of our knowledge this is a first result for this class of problems. It should be noted that the range of the parameter $p$ considered in Theorem 1.10 is larger than the range typically assumed in previous results. In fact, this is not surprising, since more regular solutions are considered. For instance, when $n=2$ the 
values of parameters $p, m, k$ can be arbitrary large, and when $n=3$ we can take $2 \leq p<5, m \leq 2, k+m \leq 5$.

- Finally, Theorem 1.13 deals with the case $p>k+m$, where we show that all weak solutions blow up in a finite time, provided the initial energy is merely negative. In addition, an upper bound for the life span of the solution is obtained (see Remark 6.1). The main idea of the proof of Theorem 1.13 is due to Georgiev and Todorova [6] and it is similar to the proofs in [6] 22. We also refer the reader to [16] for general global nonexistence theorems. As we pointed out above, the blow-up range $p>k+m$ for the parameters $p, m, k$ was first identified in [16. While [16] deals primarily with the case $k=0$ (more general equations formulated in abstract spaces) with the goal to exhibit a finite time blow-up of solutions when $p>m$, the methods used there could also handle the case $k>0$ whenever $p>k+m$. In fact, a complete proof of this phenomenon was given in [22] for the spacial case (1.3) with $m<1$. Our result formulated in Theorem 1.13 is a further generalization of the corresponding Theorem in [22], and as in [22], the proof draws substantially on the ideas introduced in [6], including the choice of the special Liapunov's function. Theorem 1.13 applies to weak solutions under the condition $p>k+m$, and in particular, it applies to the local solutions described in Corollary 1.8, Theorem 1.9 and 1.10

In conclusion, what we consider the main contribution of the paper is an existence theory for degenerate case $k>0$ that includes $m \geq 1$. In that respect, Theorem 1.6 and Theorems 1.9 1.10 provide the main technical and novel contribution of this paper. We also recall that the first global nonexistence result for the degenerate case $k>0$ was pointed out in a Remark below Theorem 4 in [16] where the authors of [16] explicitly identified the region $m+k<p$ as the region for global nonexistence.

Finally, we conclude by listing some open problems.

- Can one extract more regularity from generalized solutions? In particular, one would like to extend the range of parameters when generalized solutions are weak solutions which satisfy the variational equality (1.7).

- Uniqueness of generalized solutions is an open issue.

- Blow up of generalized solution in a finite time appears to be a challenging question.

We conclude the Introduction with a few words about the methods used for the proofs. Our method of the proof of the main result in Theorem 1.6 relies on the following:

We first establish the a priori bound for the damping-source problem under the assumption that $p \leq k+m$. This a priori bound in Section 2 allows us to construct a multi-valued fixed point argument. In order to show an existence of a fixed point, one must establish two facts.

- First, the solvability of the problem for a fixed argument (see equation (3.3)). This is accomplished by applying an appropriate Faedo-Galerkin method.

- Second, the upper semi-continuity of the nonlinear map $F$ (see Section 3 ). For this part, our argument is based on subtle approximations by weakly lower semi-continuous functions.

The remainder of this paper is devoted to the proofs of our main results. 


\section{A PRIORI BOUNDS}

We shall show that all generalized solutions admit an a priori bound in the topology specified by Definition 1.3. In addition, this a priori bound is global (i.e., it holds on $[0, T]$ for any $T>0)$ provided $p \leq k+m$.

Lemma 2.1. Let $u$ be a generalized solution of problem (1.1) with the assumption that $p \leq k+m$. Then for all initial data $u_{0} \in H_{0}^{1}(\Omega), u_{1} \in L_{2}(\Omega)$ and all $T>0$, we have the inequality

$$
|u(t)|_{1, \Omega}+\left|u_{t}(t)\right|_{0, \Omega}+\int_{0}^{t} \int_{\Omega}|u|^{k} j\left(u_{t}\right) d x d \tau \leq C_{T}\left(\left|u_{0}\right|_{1, \Omega},\left|u_{1}\right|_{0, \Omega}\right)
$$

for all $t \in[0, T]$.

If $p>k+m$ and condition (1.4) is valid, then the bound in (2.1) holds for $0 \leq t \leq T \leq T_{0}$ for some $T_{0}>0$, where $T_{0}$ may be finite and depends on the $H^{1} \times L_{2}$ norm of the initial data.

Proof. Define the following energy functions:

$$
E(t) \equiv \frac{1}{2}\left(|\nabla u(t)|_{0, \Omega}^{2}+\left|u_{t}(t)\right|_{0, \Omega}^{2}\right)
$$

and

$$
E_{1}(t) \equiv E(t)+\frac{1}{p+1} \int_{\Omega}|u(t)|^{p+1} d x
$$

By the Sobolev embedding theorem along with restriction $p+1 \leq p^{*}$ one has $\int_{\Omega}|u|^{p+1} d x \leq C\left(|u|_{1, \Omega}\right)$ along with the obvious bounds

$$
E(t) \leq E_{1}(t) \leq C(E(t))
$$

where $C(s)$ denotes throughout the proof a real-valued function which is bounded for bounded values of $s$.

By applying Definition 1.3 with $v=0$, we obtain

$$
E(t)+\int_{0}^{t} \int_{\Omega}|u|^{k} j\left(u_{t}\right) d x d \tau \leq \int_{0}^{t} \int_{\Omega}|u|^{p}\left|u_{t}\right| d x d \tau+E(0) .
$$

By adding the term

$$
\int_{0}^{t} \int_{\Omega}|u|^{p-1} u u_{t} d x d \tau=\frac{1}{p+1} \int_{\Omega}\left(|u|^{p+1}-\left|u_{0}\right|^{p+1}\right) d x
$$

to both sides of inequality (2.3) we obtain

$$
E_{1}(t)+\int_{0}^{t} \int_{\Omega}|u|^{k} j\left(u_{t}\right) d x d \tau \leq 2 \int_{0}^{t} \int_{\Omega}|u|^{p}\left|u_{t}\right| d x d \tau+E_{1}(0) .
$$

For $Q_{t} \equiv \Omega \times(0, t)$, we define

$$
Q_{A} \equiv\left\{(x, s) \in Q_{t},|u(x, s)|>1\right\} \text { and } Q_{B} \equiv\left\{(x, s) \in Q_{t},|u(x, s)| \leq 1\right\} .
$$

Then, it follows from (2.4) that

$$
E_{1}(t)+\int_{Q_{t}}|u|^{k} j\left(u_{t}\right) d Q_{t} \leq E_{1}(0)+2 \int_{Q_{A}}|u|^{p}\left|u_{t}\right| d Q_{A}+2 \int_{Q_{B}}|u|^{p}\left|u_{t}\right| d Q_{B}
$$


We estimate the integrals on the right-hand side of (2.5) as follows:

$$
\begin{aligned}
\int_{Q_{B}}|u|^{p}\left|u_{t}\right| d Q_{B} & \leq \rho\left|Q_{B}\right|+C_{\rho} \int_{Q_{B}}\left|u_{t}\right|^{2} d Q_{B} \\
& \leq \rho\left|Q_{t}\right|+C_{\rho} \int_{0}^{t} E(s) d s,
\end{aligned}
$$

where $\rho>0$ is sufficiently small and will be chosen later. Also, here and later $\left|Q_{t}\right|$ denotes the Lebesgue measure of $Q_{t}$. In order to estimate the other integral over $Q_{A}$ we choose

$$
r=\frac{p-m}{m+1}, q=\frac{m+1}{m}, \bar{q}=m+1 .
$$

If $r \leq 0$, we have

$$
\begin{aligned}
\int_{Q_{A}}|u|^{p}\left|u_{t}\right| d Q_{A} & =\int_{Q_{A}}|u|^{p-r}|u|^{r}\left|u_{t}\right| d Q_{A} \\
& \leq\left(\int_{Q_{A}}|u|^{q(p-r)} d Q_{A}\right)^{\frac{1}{q}}\left(\int_{Q_{A}}\left|u_{t}\right|^{\bar{q}} d Q_{A}\right)^{\frac{1}{q}} \\
& =\left(\int_{Q_{A}}|u|^{p+1} d Q_{A}\right)^{\frac{1}{q}}\left(\int_{Q_{A}}\left|u_{t}\right|^{m+1} d Q_{A}\right)^{\frac{1}{m+1}} .
\end{aligned}
$$

By Young's inequality, one has

$$
\begin{aligned}
\int_{Q_{A}}|u|^{p}\left|u_{t}\right| d Q_{A} & \leq C_{\epsilon} \int_{Q_{t}}|u|^{p+1} d Q_{t}+\epsilon \int_{Q_{A}}\left|u_{t}\right|^{m+1} d Q_{A} \\
& \leq C_{\epsilon} \int_{Q_{t}}|u|^{p+1} d Q_{t}+\epsilon \int_{Q_{t}}|u|^{k}\left|u_{t}\right|^{m+1} d Q_{t},
\end{aligned}
$$

where $\epsilon>0$ will be chosen later.

On the other hand if $r>0$, then we slightly modify the argument as follows:

$$
\begin{aligned}
\int_{Q_{A}}|u|^{p}\left|u_{t}\right| d Q_{A} & =\int_{Q_{A}}|u|^{p-r}|u|^{r}\left|u_{t}\right| d Q_{A} \\
& \leq\left(\int_{Q_{A}}|u|^{q(p-r)} d Q_{A}\right)^{\frac{1}{q}}\left(\int_{Q_{A}}|u|^{r \bar{q}}\left|u_{t}\right|^{\bar{q}} d Q_{A}\right)^{\frac{1}{\bar{q}}} \\
& =\left(\int_{Q_{A}}|u|^{p+1} d Q_{A}\right)^{\frac{1}{q}}\left(\int_{Q_{A}}|u|^{p-m}\left|u_{t}\right|^{m+1} d Q_{A}\right)^{\frac{1}{m+1}} .
\end{aligned}
$$

We shall first deal with the case when $p \leq m+k$. By applying Young's inequality and exploiting the assumption that $p-m \leq k$, we obtain

$$
\begin{aligned}
\int_{Q_{A}}|u|^{p}\left|u_{t}\right| d Q_{A} & \leq C_{\epsilon} \int_{Q_{A}}|u|^{p+1} d Q_{A}+\epsilon \int_{Q_{A}}|u|^{p-m}\left|u_{t}\right|^{m+1} d Q_{A} \\
& \leq C_{\epsilon} \int_{Q_{t}}|u|^{p+1} d Q_{t}+\epsilon \int_{Q_{t}}|u|^{k}\left|u_{t}\right|^{m+1} d Q_{t} .
\end{aligned}
$$

Thus, in both cases we have

$$
\begin{aligned}
\int_{Q_{t}}|u|^{p}\left|u_{t}\right| d Q_{t} & \leq \rho\left|Q_{t}\right|+C_{\rho} \int_{0}^{t} E(s) d s \\
& +\epsilon \int_{Q_{t}}|u|^{k}\left|u_{t}\right|^{m+1} d Q_{t}+C_{\epsilon} \int_{Q_{t}}|u|^{p+1} d Q_{t}
\end{aligned}
$$


where the constants $\rho, \epsilon>0$ can be taken arbitrary small. By combining inequalities (2.5) and (2.11), we obtain

$$
\begin{aligned}
E_{1}(t)+\int_{Q_{t}}|u|^{k} j\left(u_{t}\right) d Q_{t} & \leq E_{1}(0)+\epsilon \int_{Q_{t}}|u|^{k}\left|u_{t}\right|^{m+1} d Q_{t} \\
& +\rho\left|Q_{t}\right|+\left(C_{\rho}+C_{\epsilon}\right) \int_{0}^{t} E_{1}(s) d s .
\end{aligned}
$$

By taking $\epsilon$ sufficiently small and keeping in mind (2.2) along with the coercivity in Assumption 1.1, we obtain

$$
E_{1}(t)+c_{\epsilon} \int_{Q_{t}}|u|^{k} j\left(u_{t}\right) d Q_{t} \leq E_{1}(0)+\rho\left|Q_{T}\right|+C \int_{0}^{t} E_{1}(s) d s
$$

for some $c_{\epsilon}>0$. Now, by Gronwall's inequality it follows that

$$
E_{1}(t) \leq\left(E_{1}(0)+\rho\left|Q_{t}\right|\right) e^{C t} .
$$

Finally, (2.14) leads to

$$
E_{1}(t)+\int_{Q_{t}}|u|^{k} j\left(u_{t}\right) \leq C_{T}\left(E(0)+\rho\left|Q_{t}\right|\right),
$$

where the last inequality is valid for all $t \leq T$ and $T$ is being arbitrary as long as $p \leq k+m$.

If $p>k+m$, then the above bound holds locally for sufficiently small $T$. Indeed, by using Hölder's and Young's inequalities, then instead of (2.6)-(2.10) we have the following estimate:

$$
\begin{aligned}
\int_{Q_{t}}|u|^{p}\left|u_{t}\right| d Q_{t} & \leq \epsilon \int_{Q_{t}}|u|^{k}\left|u_{t}\right|^{m+1} d Q_{t}+C_{\epsilon} \int_{Q_{t}}|u|^{\frac{p(m+1)-k}{m}} d Q_{t} \\
& \leq \epsilon \int_{Q_{t}}|u|^{k}\left|u_{t}\right|^{m+1} d Q_{t}+C_{\epsilon} \int_{0}^{t}|u|_{1, \Omega}^{\frac{p(m+1)-k}{m}} d t
\end{aligned}
$$

whenever $\frac{p(m+1)-k}{m} \leq p^{*}$. If, instead, $p \leq \frac{p^{*}}{2}$, then more direct argument applies and we have

$$
\begin{aligned}
\int_{Q_{t}}|u|^{p}\left|u_{t}\right| d Q_{t} & \leq \epsilon \int_{Q_{t}}\left|u_{t}\right|^{2} d Q_{t}+C_{\epsilon} \int_{Q_{t}}|u|^{2 p} d Q_{t} \\
& \leq \epsilon \int_{Q_{t}}\left|u_{t}\right|^{2} d Q_{t}+C_{\epsilon} \int_{0}^{t}|u|_{1, \Omega}^{2 p} d t .
\end{aligned}
$$

In both cases, (2.16), (2.17) and a standard continuity argument yield the bound in (2.1) for a sufficiently small $T>0$.

\section{Fixed point ARgument And the Proof of Theorem 1.6}

Let $w \in C\left(0, T ; L_{q}(\Omega)\right)$ be a given element where throughout this section the parameter $q$ satisfies

$$
\max \left\{2 k, p+1, \min \left\{2 p, \frac{p(m+1)-k}{m}\right\}\right\}<q<p^{*} .
$$

We shall consider the following variational inequality. 
Find $u \in C_{w}\left([0, T], H_{0}^{1}(\Omega)\right) \cap C_{w}^{1}\left([0, T], L_{2}(\Omega)\right)$, with $\int_{0}^{T} \int_{\Omega}|w|^{k} j\left(u_{t}\right) d x d t<\infty$, such that for all $0<t \leq T$ the following inequality holds:

$$
\begin{aligned}
\int_{0}^{t} \int_{\Omega}\left(u_{t} v_{t}-\nabla u \nabla v\right) d x d t & +\frac{1}{2} \int_{\Omega}\left[u_{t}^{2}(t)+|\nabla u(t)|^{2}\right] d x \\
& +\int_{0}^{t} \int_{\Omega}|w|^{k}\left[j\left(u_{t}\right)-j(v)\right] d x d t \\
\leq \int_{0}^{t} \int_{\Omega}|w|^{p-1} w\left(u_{t}-v\right) d x d t+ & \frac{1}{2} \int_{\Omega}\left[u_{1}^{2}+\left|\nabla u_{0}\right|^{2}-2 u_{1} v(0)\right] d x
\end{aligned}
$$

for all test functions

$$
v \in H^{1}\left(0, t ; L_{2}(\Omega)\right) \cap L_{2}\left(0, t ; H_{0}^{1}(\Omega)\right) \cap L_{\infty}\left(Q_{t}\right), v(t)=0 .
$$

For a given argument $w \in C\left(0, T ; L_{q}(\Omega)\right)$ we consider the multi-valued mapping

$$
F: C\left(0, T ; L_{q}(\Omega)\right) \rightarrow C\left(0, T ; L_{q}(\Omega)\right),
$$

where the multi-valued action of $F$ is defined by

$$
u \in F w \text { iff } u \text { is a solution to the variational inequality (3.2). }
$$

In the next subsections we shall prove that the mapping $F$ is a well-defined multi-valued mapping on $C\left(0, T ; L_{q}(\Omega)\right)$, i.e., Range $F(w)$ is nonempty for each $w \in C\left(0, T ; L_{q}(\Omega)\right)$.

In order to establish Theorem 1.6 it suffices to show that $F$ has a fixed point. We accomplish this by using Kakutani-type Theorem [34] and the a priori bound established in Lemma 2.1 However, we first need to prove the following facts:

- $F(K)$ is convex and compact on $C\left(0, T ; L_{q}(\Omega)\right)$, where $K$ is being a suitably chosen (large) ball in $C\left(0, T ; L_{q}(\Omega)\right)$ whose radius depends on the initial data.

- $F$ is upper semi-continuous. It is important to note here that due to the compactness of $F(K)$, proving the upper semi-continuity of $F$ amounts to showing the following statement:

for a given sequence $w_{n} \rightarrow w$ in $C\left(0, T ; L_{q}(\Omega)\right)$, and $u_{n} \rightarrow u$ in $C\left(0, T ; L_{q}(\Omega)\right)$ where $u_{n} \in F\left(w_{n}\right)$, then we have $u \in F(w)$. Indeed, this is equivalent to the fact that the graph of $F$ is closed in $C\left([0, T] ; L_{q}(\Omega)\right) \times C\left([0, T] ; L_{q}(\Omega)\right)$.

3.1. Well-posedness of the map $F$. For a given function $w \in C\left(0, T ; L_{q}(\Omega)\right)$, we consider the equation

$$
u_{t t}-\Delta u+|w|^{k} \partial j\left(u_{t}\right)=|w|^{p-1} w,
$$

whose variational formulation is the following: find

$$
u \in C\left([0, T], H_{0}^{1}(\Omega)\right) \cap C_{w}^{1}\left([0, T], L_{2}(\Omega)\right), \quad u_{t t} \in L_{2}\left(0, T ; H^{-1}(\Omega)\right)
$$

such that the following identity holds:

$$
\begin{array}{r}
\int_{0}^{t} \int_{\Omega}\left[u_{t t} v+\nabla u \nabla v\right] d x d t+\int_{0}^{t} \int_{\Omega}|w|^{k} \partial j\left(u_{t}\right) v d x d t \\
=\int_{0}^{t} \int_{\Omega}|w|^{p-1} w v d x d t \\
u(0)=u_{0}, u_{t}(0)=u_{1},
\end{array}
$$

for all test functions $v \in C_{w}\left([0, T] ; H_{0}^{1}(\Omega) \cap L_{(m+1) \frac{q}{q-k}}(\Omega)\right)$. 
The main results in this subsection are the following.

Lemma 3.1. Assume the validity of Assumption 1.1 and condition (3.1). Then, there exists a unique solution $u$ to the variational identity (3.4) such that $u \in$ $C\left(0, T ; H_{0}^{1}(\Omega)\right) \cap C^{1}\left(0, T ; L_{2}(\Omega)\right)$ and the following bound holds for all $t \leq T$ :

$$
\begin{aligned}
|u(t)|_{1, \Omega}^{2} & +\left|u_{t}(t)\right|_{0, \Omega}^{2}+\int_{Q_{t}}|w|^{k}\left|u_{t}\right|^{m+1} d Q_{t} \\
& \leq C_{T}\left(\left|u_{0}\right|_{1, \Omega},\left|u_{1}\right|_{0, \Omega},|w|_{C\left([0, T] ; L_{q}(\Omega)\right)}\right) .
\end{aligned}
$$

In addition, the following energy identity holds:

$$
\begin{aligned}
& \frac{1}{2}\left(|\nabla u(t)|_{0, \Omega}^{2}+\left|u_{t}(t)\right|_{0, \Omega}^{2}\right)+\int_{Q_{t}}|w|^{k} \partial j\left(u_{t}\right) u_{t} d Q_{t} \\
& \quad=\frac{1}{2}\left(|\nabla u(0)|_{0, \Omega}^{2}+\left|u_{t}(0)\right|_{0, \Omega}^{2}\right)+\int_{Q_{t}}|w|^{p-1} w u_{t} d Q_{t}
\end{aligned}
$$

Corollary 3.2. For each fixed $w \in C\left([0, T], L_{q}(\Omega)\right), F(w) \neq \emptyset$. Moreover, one has $C\left([0, T], L_{q}(\Omega)\right) \subset$ Dom $F$.

Proof of Lemma 3.1. We consider a standard Galerkin approximation scheme for the solution of (3.4) based on the eigenfunctions $\left\{e_{k}\right\}_{k=1}^{\infty}$ of the operator $A=-\triangle$ with zero boundary condition on $\partial \Omega$. That is, we let $u_{n}(t)=\sum_{k=1}^{n} u_{n, k}(t) e_{k}$, where $u_{n}(t)$ satisfies

$$
\begin{array}{r}
\left(u_{n t t}, v\right)_{\Omega}+\left(\nabla u_{n}, \nabla v\right)_{\Omega}+\left(|w|^{k} \partial j\left(u_{n t}\right), v\right)_{\Omega}=\left(|w|^{p-1} w, v\right)_{\Omega} \\
\left(u_{n}(0), v\right)_{\Omega}=\left(u_{0}, v\right)_{\Omega}, \quad\left(u_{n t}(0), v\right)_{\Omega}=\left(u_{1}, v\right)_{\Omega}
\end{array}
$$

for all $v \in V_{n}:=$ the linear span of $\left\{e_{1}, \ldots, e_{n}\right\}$, and for convenience, we use $(., .)_{\Omega}$ to denote the standard $L_{2}(\Omega)$-inner product.

By standard nonlinear ordinary differential equations theory one obtains the existence of a global solution to (3.7) with the following a priori bounds which are uniform in $n$ :

$$
\begin{aligned}
& \frac{1}{2}\left(\left|\nabla u_{n}(t)\right|_{0, \Omega}^{2}+\left|u_{n t}(t)\right|_{0, \Omega}^{2}\right)+\int_{Q_{t}}|w|^{k} \partial j\left(u_{n t}\right) u_{n t} d Q_{t} \\
& =\frac{1}{2}\left(\left|\nabla u_{n}(0)\right|_{0, \Omega}^{2}+\left|u_{n t}(0)\right|_{0, \Omega}^{2}\right)+\int_{Q_{t}}|w|^{p-1} w u_{n t} d Q_{t} .
\end{aligned}
$$

By using the restrictions imposed on the parameter $q$, we obtain the estimates

$$
\begin{aligned}
\int_{Q_{t}}|w|^{p}\left|u_{n t}\right| d Q_{t} & \leq \epsilon \int_{Q_{t}}\left|u_{n t}\right|^{m+1}|w|^{k} d Q_{t}+C_{\epsilon} \int_{Q_{t}}|w|^{\frac{p(m+1)-k}{m}} d Q_{t} \\
& \leq \epsilon \int_{Q_{t}}\left|u_{n t}\right|^{m+1}|w|^{k} d Q_{t}+C_{\epsilon} \int_{0}^{t}|w|_{L_{q}(\Omega)}^{\frac{p(m+1)-k}{m}} d t
\end{aligned}
$$

when $\frac{p(m+1)-k}{m} \leq q$, and

$$
\begin{aligned}
\int_{Q_{t}}|w|^{p}\left|u_{n t}\right| d Q_{t} & \leq \epsilon \int_{Q_{t}}\left|u_{n t}\right|^{2} d Q_{t}+C_{\epsilon} \int_{Q_{t}}|w|^{2 p} d Q_{t} \\
& \leq \epsilon \int_{Q_{t}}\left|u_{n t}\right|^{2} d Q_{t}+C_{\epsilon} \int_{0}^{t}|w|_{L_{q}(\Omega)}^{2 p} d t
\end{aligned}
$$


when $2 p \leq q$. Thus, it follows from (3.9), (3.10) and (3.8) that

$$
\begin{gathered}
\left|u_{n}(t)\right|_{1, \Omega}^{2}+\left|u_{n t}(t)\right|_{0, \Omega}^{2}+\int_{Q_{t}}|w|^{k} j\left(u_{n t}\right) d Q_{t} \\
\leq C_{T}\left(\left|u_{0}\right|_{1, \Omega},\left|u_{1}\right|_{0, \Omega},|w|_{C\left([0, T] ; L_{q}(\Omega)\right)}\right) .
\end{gathered}
$$

By using the coercivity condition in Assumption 1.1, we obtain

$$
\begin{array}{r}
\left|u_{n}(t)\right|_{1, \Omega}^{2}+\left|u_{n t}(t)\right|_{0, \Omega}^{2}+\int_{Q_{T}}|w|^{k}\left|u_{n t}\right|^{m+1} d Q_{T} \\
\leq C_{T}\left(\left|u_{0}\right|_{1, \Omega},\left|u_{1}\right|_{0, \Omega},|w|_{C\left([0, T] ; L_{q}(\Omega)\right)}\right) .
\end{array}
$$

Hence, there exists a subsequence of $\left\{u_{n}\right\}$, which we still denote by $\left\{u_{n}\right\}$, that satisfies

$$
\begin{aligned}
u_{n} & \rightarrow \text { u weakly* in } L_{\infty}\left(0, T ; H^{1}(\Omega)\right), \\
u_{n t} & \rightarrow u_{t} \text { weakly }^{*} \text { in } L_{\infty}\left(0, T ; L_{2}(\Omega)\right) .
\end{aligned}
$$

Now, consider two solutions $u_{n}$ and $u_{l}$, where without loss of generality we assume $l \geq n$. Denote $U_{n l} \equiv u_{n}-u_{l}$. Then, it follows from (3.4) that $U_{n l}$ satisfies variational equality

$$
\begin{aligned}
& \left(U_{n l t t}, v\right)_{\Omega}+\left(\nabla U_{n l}, \nabla v\right)_{\Omega}+\left(|w|^{k} \partial j\left(U_{n t}\right)-\partial j\left(U_{l t}\right), v\right)_{\Omega}=0, \\
& \left(U_{n l}(0), v\right)_{\Omega}=\left(u_{n 0}-u_{l 0}, v\right)_{\Omega},\left(u_{n l t}(0), v\right)_{\Omega}=\left(u_{1 n}-u_{1 l}, v\right)_{\Omega},
\end{aligned}
$$

for all $v \in V_{n}$.

By setting $v=U_{n l t}$ in (3.14) and by using the strong convergence of the approximations to the initial data, one easily obtains the following convergence result:

$$
\left|U_{n l t}(t)\right|_{0, \Omega}^{2}+\left|U_{n l}(t)\right|_{1, \Omega}^{2}+\int_{0}^{t}|w|^{k}\left(\partial j\left(u_{n t}\right)-\partial j\left(u_{l t}\right), U_{n l t}\right)_{\Omega} d s \rightarrow 0,
$$

as $n, l \rightarrow \infty$.

Now from (3.15) and the strong coercivity assumption, we conclude

$$
\left|U_{n l t}(t)\right|_{0, \Omega}^{2}+\left|U_{n l}(t)\right|_{1, \Omega}^{2}+\int_{0}^{t} \int_{\Omega}|w|^{k}\left|U_{n l t}\right|^{m+1} d x d s \rightarrow 0,
$$

as $n, l \rightarrow \infty$. From (3.16) we infer the strong convergence

$$
|w|^{\frac{k}{m+1}} u_{n t} \rightarrow \eta \text { in } L_{m+1}\left(Q_{T}\right), \text { as } n \rightarrow \infty,
$$

for some $\eta \in L_{m+1}\left(Q_{T}\right)$. Moreover, we have

$$
\begin{aligned}
u_{t n} & \rightarrow u_{t} \text { strongly in } L_{\infty}\left(0, T ; L_{2}(\Omega)\right), \\
u_{n} & \rightarrow u \text { strongly in } L_{\infty}\left(0, T ; H_{0}^{1}(\Omega)\right) .
\end{aligned}
$$

Here we remark that the above strong convergence allows us to reconstruct the limit function $\eta$. Indeed,

Thus,

$$
\eta=|w|^{\frac{k}{m+1}} u_{t} .
$$

$$
|w|^{\frac{k}{m+1}} u_{n t} \rightarrow|w|^{\frac{k}{m+1}} u_{t} \text { strongly in } L_{m+1}\left(Q_{T}\right) \text {, as } n \rightarrow \infty .
$$

Equivalently,

$$
\int_{Q_{T}}|w|^{k}\left|u_{t n}-u_{t}\right|^{m+1} d Q_{T} \rightarrow 0, \text { as } n \rightarrow \infty .
$$


In particular, as $n \rightarrow \infty$ (passing to a subsequence if necessary),

$$
|w|^{k} u_{n t} \rightarrow|w|^{k} u_{t} \text { point-wise almost everywhere } x, t \in Q_{T} .
$$

Now in order to pass to the limit in the nonlinear term, we shall prove

$$
|w|^{\frac{k m}{m+1}} \partial j\left(u_{n t}\right) \rightarrow|w|^{\frac{k m}{m+1}} \partial j\left(u_{t}\right) \text { weakly in } L_{\frac{m+1}{m}}\left(Q_{T}\right) \text {, as } n \rightarrow \infty \text {. }
$$

To see this, we recall the a priori bound in (3.12), which implies

$$
\int_{Q_{T}}\left[|w|^{\frac{k m}{m+1}}\left|u_{n t}\right|^{m}\right]^{\frac{m+1}{m}} d Q_{T}=\int_{Q_{T}}|w|^{k}\left|u_{n t}\right|^{m+1} d Q_{T} \leq C_{T} .
$$

Also, the growth condition imposed on $\partial j(s)$ yields

$$
\int_{Q_{T}}\left[|w|^{\frac{k m}{m+1}}\left|\partial j\left(u_{n t}\right)\right|\right]^{\frac{m+1}{m}} d Q_{T} \leq M_{T}
$$

for some constant $M_{T}>0$. Hence, by passing to a subsequence if necessary, one has

and

$$
|w|^{\frac{k m}{m+1}}\left|u_{n t}\right|^{m} \rightarrow l \text { weakly in } L_{\frac{m+1}{m}}\left(Q_{T}\right)
$$

$$
|w|^{\frac{k m}{m+1}} \partial j\left(u_{n t}\right) \rightarrow J \text { weakly in } L_{\frac{m+1}{m}}\left(Q_{T}\right) .
$$

By appealing to the almost everywhere point-wise convergence in (3.20) and the piecewise continuity of $\partial j(s)$, we can identify the limits $l$ and $J$. Indeed,

$$
l=|w|^{\frac{k m}{m+1}} u_{t}^{m}, \quad J=|w|^{\frac{k m}{m+1}} \partial j\left(u_{t}\right) .
$$

Therefore, we have

$$
|w|^{\frac{k m}{m+1}}\left|u_{n t}\right|^{m} \rightarrow|w|^{\frac{k m}{m+1}}\left|u_{t}\right|^{m} \text { weakly in } L_{\frac{m+1}{m}}\left(Q_{T}\right)
$$

and

$$
|w|^{\frac{k m}{m+1}} \partial j\left(u_{n t}\right) \rightarrow|w|^{\frac{k m}{m+1}} \partial j\left(u_{t}\right) \text { weakly in } L_{\frac{m+1}{m}}\left(Q_{T}\right),
$$

as desired in (3.21).

From the weak convergence in (3.21) and the strong convergence in (3.19) we infer that

$$
\int_{Q_{t}}|w|^{k} \partial j\left(u_{n t}\right) u_{n t} d Q_{t} \rightarrow \int_{Q_{t}}|w|^{k} \partial j\left(u_{t}\right) u_{t} d Q_{t} .
$$

Indeed, to see (3.24) we write

$$
\int_{Q_{t}}|w|^{k} \partial j\left(u_{t n}\right) u_{n t} d Q_{t}=\int_{Q_{t}}|w|^{\frac{k m}{m+1}} \partial j\left(u_{n t}\right)|w|^{\frac{k}{m+1}} u_{n t} d Q_{t},
$$

and thus (3.24) follows easily from (3.21), (3.19) and duality.

Our next step is to establish the following convergence:

$$
\int_{Q_{t}}|w|^{k}\left|u_{n t}\right|^{m+1} d x d s \rightarrow \int_{Q_{t}}|w|^{k}\left|u_{t}\right|^{m+1} d x d s .
$$

Indeed, (3.25) becomes clear after writing

$$
|w|^{k}\left|u_{n t}\right|^{m+1}=|w|^{\frac{k m}{m+1}}\left|u_{n t}\right|^{m-1} u_{n t}|w|^{\frac{k}{m+1}} u_{n t},:=g\left(z_{n}\right) z_{n},
$$

where we have used the notation

$$
z_{n} \equiv|w|^{\frac{k}{m+1}} u_{n t}, g\left(z_{n}\right) \equiv\left|z_{n}\right|^{m-1} z_{n} .
$$


Let $z=|w|^{\frac{k}{m+1}} u_{t}$. Then, we note that (3.19) and (3.22) yield

$$
\begin{gathered}
z_{n} \rightarrow z \text { strongly in } L_{m+1}\left(Q_{T}\right), \\
g\left(z_{n}\right) \rightarrow g(z) \text { weakly in } L_{\frac{m+1}{m}}\left(Q_{T}\right) .
\end{gathered}
$$

Therefore, (3.25) follows easily from (3.26), the convergence in (3.27) and duality.

By applying the convergence in (3.25) to inequality (3.12) and keeping in mind weak lower semi-continuity of the norm, we obtain

$$
\begin{gathered}
|u(t)|_{1, \Omega}^{2}+\left|u_{t}(t)\right|_{0, \Omega}^{2}+\int_{Q_{t}}|w|^{k}\left|u_{t}\right|^{m+1} d Q_{t} \\
\leq C_{T}\left(\left|u_{0}\right|_{1, \Omega},\left|u_{1}\right|_{0, \Omega},|w|_{C\left(0, T ; L_{q}(\Omega)\right)}\right),
\end{gathered}
$$

which establishes (3.5). We shall next prove that

$$
\int_{Q_{t}}|w|^{p-1} w u_{n t} d Q_{t} \rightarrow \int_{Q_{t}}|w|^{p-1} w u_{t} d Q_{t}
$$

When $2 p \leq q$ the above is just a consequence of the $L_{2}$-weak convergence of $u_{n t}$ and the fact that $|w|^{p} \in L_{2}\left(Q_{t}\right)$. Otherwise, if $\frac{p(m+1)-k}{m} \leq q$, then more effort is needed:

$$
\begin{array}{r}
\left.\left.\int_{Q_{t}}|| w\right|^{p-1} w\left(u_{n t}-u_{t}\right)\left|d Q_{t} \leq \int_{Q_{t}}\right| w\right|^{p}\left|u_{n t}-u_{t}\right| d Q_{t} \\
\leq \epsilon \int_{Q_{t} \cap\{|w| \geq 1\}}|w|^{\frac{p(m+1)-k}{m}} d Q_{t}+\epsilon\left|Q_{t}\right|+C_{\epsilon} \int_{Q_{t}}|w|^{k}\left|u_{n t}-u_{t}\right|^{m+1} d Q_{t} \\
\leq \epsilon \int_{0}^{t}\|w\|_{L_{q}(\Omega)}^{\frac{p(m+1)-k}{m}} d t+\epsilon\left|Q_{t}\right|+C_{\epsilon} \int_{Q_{t}}|w|^{k}\left|u_{n t}-u_{t}\right|^{m+1} d Q_{t} .
\end{array}
$$

By (3.19) we conclude that the last term above converges to zero as $n \rightarrow \infty$. Thus letting $\epsilon \rightarrow 0$, we obtain the desired conclusion in (3.29). By using (3.18), (3.24), (3.29) and the energy identity in (3.8), we obtain the energy identity (3.6).

Our final step is the passage to the limit in the variational form of the equation. By first taking a test function $v$ as smooth as necessary we obtain

$$
\begin{aligned}
\left(|w|^{k} \partial j\left(u_{n t}\right), v\right)_{Q_{T}} & =\left(|w|^{\frac{k m}{m+1}} \partial j\left(u_{n t}\right),|w|^{\frac{k}{m+1}} v\right)_{Q_{T}} \\
& \rightarrow\left(|w|^{\frac{k m}{m+1}} \partial j\left(u_{t}\right),|w|^{\frac{k}{m+1}} v\right)_{Q_{T}}
\end{aligned}
$$

as long as $v|w|^{\frac{k}{m+1}} \in L_{m+1}\left(Q_{T}\right)$. Indeed, the latter holds for $v \in L_{q(m+1) /(q-k)}$, as desired. The passage to the limit in the linear terms is standard, and thus it is omitted.

\subsection{Proof of Corollary 3.2.}

Proof. The statement in the corollary follows once we show that the unique solution $u$ constructed in Lemma 3.1 belongs to $F(w)$. Note that the necessary regularity of $u$ follows from the statement in Lemma 3.1 
On the other hand, by adding the energy identity in (3.6) to the variational formulation in (3.4), we obtain that $u$ satisfies

$$
\begin{array}{r}
\int_{0}^{t} \int_{\Omega}\left(u_{t} v_{t}-\nabla u \nabla v\right) d x d t+1 / 2 \int_{\Omega}\left[u_{t}^{2}(t)+|\nabla u(t)|^{2}\right] d x \\
+\int_{0}^{t} \int_{\Omega}|w|^{k} \partial j\left(u_{t}\right)\left(u_{t}-v\right) d x d t \\
(3.32)=\int_{0}^{t} \int_{\Omega}|w|^{p-1} w\left(u_{t}-v\right) d x d t+1 / 2 \int_{\Omega}\left[u_{1}^{2}+\left|\nabla u_{0}\right|^{2}-2 u_{1} v(0)\right] d x,
\end{array}
$$

for all test functions

$$
v \in H^{1}\left(0, t ; L_{2}(\Omega)\right) \cap L_{2}\left(0, t ; H_{0}^{1}(\Omega)\right) \cap L_{\infty}\left(Q_{t}\right), v(t)=0 .
$$

By recalling the inequality

$$
\partial j\left(u_{t}\right)\left(u_{t}-v\right) \geq j\left(u_{t}\right)-j(v)
$$

leads to the desired inequality in (3.2) satisfied by the solution $u$ that corresponds to the element $w$.

3.3. Compactness and convexity of $F$. Let $K$ be a ball in $C\left([0, T] ; L_{q}(\Omega)\right)$. Then, we have:

Lemma 3.3. $F(K)$ is compact and $F(w)$ is convex for every $w \in K$.

Proof. By setting $v=0$ in the variational inequality (3.2) we easily see that any solution $u$ to this variational inequality with $w \in K$ and with initial data of finite energy must satisfy

$$
|u(t)|_{1, \Omega}+\left|u_{t}(t)\right|_{0, \Omega} \leq C\left(|w|_{C\left([0, T] ; L_{q}(\Omega)\right)},\left|u_{0}\right|_{1, \Omega},\left|u_{1}\right|_{0, \Omega}\right) .
$$

The above inequality and Simon's compactness lemma [28] imply the compactness of $F(K)$.

The convexity of $F(w)$ follows from the definition given by (3.2) and the convexity of $j(s)$.

3.4. Upper semi-continuity of $F$. Before proving the upper semi-continuity of the mapping $F$, we shall prove the following proposition which is central to the argument.

Proposition 3.4. Let $u_{n t}$ be any sequence which converges weakly in $L_{2}\left(Q_{T}\right)$ to a function $u_{t}$. Let $w_{n} \rightarrow w$ in $C\left([0, T] ; L_{q}(\Omega)\right)$, where $q$ satisfies (3.1). Further assume that $\left\|\left|w_{n}\right|^{k}\left|u_{n t}\right|^{m+1}\right\|_{L_{1}\left(Q_{T}\right)} \leq M$ uniformly in $n$. Then, we have the following:

$$
\begin{array}{r}
\int_{Q_{t}}|w|^{k} j\left(u_{t}\right) d Q_{t} \leq \liminf _{n \rightarrow \infty} \int_{Q_{t}}\left|w_{n}\right|^{k} j\left(u_{n t}\right) d Q_{t}, \\
(3.34) \int_{Q_{t}}\left|w_{n}\right|^{p-1} w_{n}\left(u_{n t}-v\right) d Q_{t} \rightarrow \int_{Q_{t}}|w|^{p-1} w\left(u_{t}-v\right) d Q_{t}, \text { as } n \rightarrow \infty, \\
\int_{Q_{t}}\left|w_{n}\right|^{k} j(v) d Q_{t} \rightarrow \int_{Q_{t}}|w|^{k} j(v) d Q_{t} \text {, as } n \rightarrow \infty,
\end{array}
$$

for all $v \in L_{\infty}\left(Q_{t}\right)$. 
Proof. The second part of Proposition 3.4 in the case when $p \leq \frac{q}{2}$, follows directly from the strong convergence

$$
\left|w_{n}\right|^{p-1} w_{n} \rightarrow|w|^{p-1} w \text { in } L_{2}\left(Q_{t}\right)
$$

and the weak convergence

$$
u_{n t} \rightarrow u_{t} \text { weakly in } L_{2}\left(Q_{t}\right) .
$$

If, instead, $p(m+1)-k<q m$ (see condition (3.1)) we have

$$
\begin{array}{r}
w_{n}^{\frac{k}{m+1}} u_{n t} \rightarrow w^{\frac{k}{m+1}} u_{t} \text { weakly in } L_{m+1}\left(Q_{T}\right), \\
\left|w_{n}\right|^{p-1-\frac{k}{m+1}} w_{n} \rightarrow|w|^{p-1-\frac{k}{m+1}} w \text { strongly in } L_{\frac{m+1}{m}}\left(Q_{T}\right) .
\end{array}
$$

Indeed, the second assertion follows from the strong convergence of $w_{n}$ in $L_{q}\left(Q_{T}\right)$ and the restriction $\left(p-\frac{k}{m+1}\right) \frac{m+1}{m} \leq q$, implied by (3.1). As for the second statement in (3.35), we notice first that by the assumption imposed in Proposition 3.4 then $\left|w_{n}\right|^{\frac{k}{m+1}} u_{n t}$ is uniformly bounded in $L_{m+1}\left(Q_{T}\right)$. Hence, $\left|w_{n}\right|^{\frac{k}{m+1}} u_{n t} \rightarrow \eta$ weakly in $L_{m+1}\left(Q_{T}\right)$. On the other hand, by using the weak convergence of $u_{n t}$ in $L_{2}\left(Q_{T}\right)$ and the strong convergence $w_{n}^{\frac{k}{m+1}} \rightarrow w^{\frac{k}{m+1}}$ in $L_{2}\left(Q_{T}\right)$ (note that by (3.1) $\frac{k}{m+1}<\frac{q}{2}$ ) we obtain

$$
\left|w_{n}\right|^{\frac{k}{m+1}} u_{n t} \rightarrow|w|^{\frac{k}{m+1}} u_{t} \text { weakly in } L_{1}\left(Q_{T}\right) .
$$

This allows us to identify $\eta$ with $\eta=w^{\frac{k}{m+1}} u_{t}$, as desired.

Having established (3.35) the rest of the argument is straightforward. It suffices to write

$$
\int_{Q_{t}}\left|w_{n}\right|^{p-1} w_{n} u_{n t} d Q_{t}=\int_{Q_{t}}\left(\left|w_{n}\right|^{\frac{k}{m+1}} u_{n t}\right)\left(\left|w_{n}\right|^{p-1-\frac{k}{m+1}} w_{n}\right) d Q_{t},
$$

where the first bracket in the right-hand side of (3.36) converges weakly in $L_{m+1}\left(Q_{t}\right)$, and the second bracket converges strongly in $L_{\frac{m+1}{m}}\left(Q_{t}\right)$. This completes the proof of convergence

$$
\int_{Q_{t}}\left|w_{n}\right|^{p-1} w_{n} u_{n t} d Q_{t} \rightarrow \int_{Q_{t}}|w|^{p-1} w u_{t} d Q_{t},
$$

and hence the second convergence in (3.34) follows.

The third part in the proposition is straightforward, and it follows from the strong convergence of $w_{n}$ in $L_{\frac{2}{k}}\left(Q_{T}\right)$, which is implied by the assumption $k \leq q$. To complete the proof of Proposition 3.4 we need to prove the first part. To accomplish this, we introduce the following approximation (truncation) of $j$ :

$$
j_{N}(s) \equiv\left\{\begin{array}{l}
j(s), \quad|s| \leq N, \\
j(N)+\partial j(N)(s-N), \quad s>N, \\
j(-N)+\partial j(-N)(s+N), \quad s<-N .
\end{array}\right.
$$

It is easy to see that for each $N, J_{N}$ is convex, continuous, and satisfies

$$
\begin{aligned}
& j_{N}(s) \leq j(s), \\
& j_{N}(s) \rightarrow j(s), \text { as } N \rightarrow \infty, \quad \text { for all } s \in \mathbb{R} .
\end{aligned}
$$

Moreover, $\partial j_{N}(s)=\partial j(s)$, for all $s \in[-N, N], \partial j_{N}(s)=\partial j(N)$, for all $s \geq N$ and $\partial j_{N}(s)=\partial j(-N)$, for all $s \leq-N$. Hence, for all $v \in L_{2}\left(Q_{t}\right)$, we have

$$
j_{N}(v) \in L_{2}\left(Q_{t}\right), \partial j_{N}(v) \in L_{\infty}\left(Q_{t}\right) .
$$


In what follows we shall assume, without loss of generality, that $j \geq 0$. Then, from (3.37) we infer that for each fixed $N$

$$
\liminf _{n \rightarrow \infty} \int_{Q_{t}}\left|w_{n}\right|^{k} j\left(u_{n t}\right) d x d t \geq \liminf _{n \rightarrow \infty} \int_{Q_{t}}\left|w_{n}\right|^{k} j_{N}\left(u_{n t}\right) d x d t .
$$

From the definition of a sub-gradient we recall that

$$
j_{N}(v) \leq j_{N}\left(u_{n t}(t, x)\right)+\partial j_{N}(v)\left(v-u_{n t}(t, x)\right), \text { for all } v \in \mathbb{R} .
$$

By recalling (3.38), it then follows from 3.40) that

$$
\int_{Q_{t}}\left|w_{n}\right|^{k}\left[j_{N}(v)-\partial j_{N}(v)\left(v-u_{n t}\right)\right] d x d t \leq \int_{Q_{t}}\left|w_{n}\right|^{k} j_{N}\left(u_{n t}\right) d x d t,
$$

for all $v \in L_{2}\left(Q_{t}\right)$, and from (3.37) one has

$$
\int_{Q_{t}}\left|w_{n}\right|^{k}\left[j_{N}(v)-\partial j_{N}(v)\left(v-u_{n t}\right)\right] d x d t \leq \int_{Q_{t}}\left|w_{n}\right|^{k} j\left(u_{n t}\right) d x d t,
$$

for all $v \in L_{2}\left(Q_{t}\right)$. By noting that

$$
\left|w_{n}\right|^{k} u_{n t} \rightarrow|w|^{k} u_{t} \text { weakly in } L_{r}\left(Q_{t}\right) \text {, for some } r>1 \text {, as } n \rightarrow \infty \text {, and }
$$

$$
\int_{Q_{t}}\left|w_{n}\right|^{k} j_{N}(v) d x d t \rightarrow \int_{Q_{t}}|w|^{k} j_{N}(v) d x d t \text {, as } n \rightarrow \infty,
$$

and by recalling the fact that $\partial j_{N}(v) \in L_{\infty}\left(Q_{t}\right)$, we obtain

$$
\int_{Q_{t}}|w|^{k}\left[j_{N}(v)-\partial j_{N}(v)\left(v-u_{t}\right)\right] d x d t \leq \liminf _{n \rightarrow \infty} \int_{Q_{t}}\left|w_{n}\right|^{k} j\left(u_{n t}\right) d x d t,
$$

for all $v \in L_{2}\left(Q_{t}\right)$. By taking $v=u_{t} \in L_{2}\left(Q_{t}\right)$, then 3.44 yields

$$
\int_{Q_{t}}|w|^{k} j_{N}\left(u_{t}\right) d x d t \leq \liminf _{n \rightarrow \infty} \int_{Q_{t}}\left|w_{n}\right|^{k} j\left(u_{n t}\right) d x d t .
$$

Since $j_{N}\left(u_{t}\right) \rightarrow j\left(u_{t}\right)$ almost everywhere in $Q_{t}$ as $N \rightarrow \infty$, and $j_{N}(s)$ is nonnegative for each $N$, then we are in a position to apply Fatou's Lemma and are able to conclude

$$
\int_{Q_{t}}|w|^{k} j\left(u_{t}\right) d x d t \leq \liminf _{n \rightarrow \infty} \int_{Q_{t}}\left|w_{n}\right|^{k} j\left(u_{n t}\right) d x d t .
$$

Hence, the first part in Proposition 3.4 follows immediately, which completes the proof.

Remark 3.5. In the special case when $j(s)=|s|^{m+1}$, a more direct proof of the first part of Proposition 3.4 can be given. To see this, we introduce an auxiliary variable

$$
z_{n} \equiv\left|w_{n}\right|^{\frac{k}{m+1}} u_{n t}
$$

In particular, since $w_{n} \rightarrow w$ in $C\left([0, T] ; L_{q}(\Omega)\right)$, we have

$$
\left|w_{n}\right|^{\frac{k}{m+1}} \rightarrow|w|^{\frac{k}{m+1}} \text { strongly in } L_{\frac{q(m+1)}{k}}(\Omega),
$$

where $\frac{q(m+1)}{k}>2$, since $q \geq 2 k$. Thus, by the virtue of the weak convergence in $L_{2}\left(Q_{t}\right)$ of $u_{n t}$ we infer

$$
z_{n} \rightarrow z \text { weakly in } L_{r}\left(Q_{t}\right) \text {, for some } r>1 \text {, }
$$

where

$$
z=|w|^{\frac{k}{m+1}} u_{t}
$$


In particular, $\left\{z_{n}\right\}$ is weakly compact on $L_{1}\left(Q_{t}\right)$ and $\left\{z_{n}\right\}$ (or passing to a subsequence) converges in $L_{1}\left(Q_{t}\right)$.

On the other hand, the function $z \mapsto \int_{Q_{t}} j(z) d Q_{t}$ is weakly lower semi-continuous on $L_{1}\left(Q_{t}\right)$ (see [3], Prop 2.10, page 67). Therefore,

$$
\int_{Q_{t}} j(z) d Q_{t} \leq \liminf _{n \rightarrow \infty} \int_{Q_{t}} j\left(z_{n}\right) d Q_{t},
$$

or equivalently,

$$
\int_{Q_{t}}|w|^{k}\left|u_{t}\right|^{m+1} d Q_{t} \leq \liminf _{n \rightarrow \infty} \int_{Q_{t}}\left|w_{n}\right|^{k}\left|u_{n t}\right|^{m+1} d Q_{t},
$$

as desired.

We are now in a position to prove the upper semi-continuity of the mapping $F$. Specifically, we have the following lemma.

Lemma 3.6. Let $w_{n} \rightarrow w$ in $C\left([0, T] ; L_{q}(\Omega)\right)$. Let $u_{n} \in F\left(w_{n}\right)$ be such that $u_{n} \rightarrow u$ in $C\left([0, T] ; L_{q}(\Omega)\right)$. Then, $u \in F(w)$.

Proof. Since $u_{n} \in F\left(w_{n}\right)$, then from the definition of the mapping $F$ we have the following a priori bounds:

$$
\begin{aligned}
\left|u_{n}(t)\right|_{1, \Omega}+\left|u_{n t}(t)\right|_{0, \Omega} & \leq C\left(|w|_{C\left([0, T] ; L_{q}(\Omega)\right)},\left|u_{0}\right|_{1 . \Omega},\left|u_{1}\right|_{0, \Omega}\right), \\
\int_{Q_{T}}\left|w_{n}\right|^{k} j\left(u_{n t}\right) d Q_{T} & \leq C\left(|w|_{C\left([0, T] ; L_{q}(\Omega)\right)},\left|u_{0}\right|_{1 . \Omega},\left|u_{1}\right|_{0, \Omega}\right) .
\end{aligned}
$$

Therefore, by passing to a subsequence if necessary we have

$$
\begin{aligned}
u_{n} & \rightarrow u \text { weakly* in } L_{\infty}\left(0, T ; H^{1}(\Omega)\right), \\
u_{n t} & \rightarrow u_{t} \text { weakly }^{*} \text { in } L_{\infty}\left(0, T ; L_{2}(\Omega)\right) .
\end{aligned}
$$

By Simon's compactness criterion and recalling that $q<\frac{2 n}{n-2}$ we conclude that

$$
u_{n} \rightarrow u \text {, strongly in } C\left([0, T] ; L_{q}(\Omega)\right) \text {. }
$$

Therefore, the proof of the lemma will be completed if we show that $u \in F(w)$. In order to do so, we recall the variational definition of the mapping $F$ given in (3.2). Since $u_{n} \in F\left(w_{n}\right)$, we have

$$
\begin{aligned}
\int_{0}^{t} \int_{\Omega}\left(u_{n t} v_{t}-\nabla u_{n} \nabla v\right) d x d t & +1 / 2 \int_{\Omega}\left[\left|u_{n t}(t)\right|^{2}+\left|\nabla u_{n}(t)\right|^{2}\right] d x \\
& +\int_{0}^{t} \int_{\Omega}\left|w_{n}\right|^{k}\left[j\left(u_{n t}\right)-j(v)\right] d x d t \\
\leq \int_{0}^{t} \int_{\Omega}\left|w_{n}\right|^{p-1} w_{n}\left(u_{n t}-v\right) d x d t & +1 / 2 \int_{\Omega}\left[u_{1}^{2}+\left|\nabla u_{0}\right|^{2}-2 u_{1} v(0)\right] d x,
\end{aligned}
$$

for all test functions

$$
v \in H^{1}\left(0, t ; L_{2}(\Omega)\right) \cap L_{2}\left(0, t ; H_{0}^{1}(\Omega)\right) \cap L_{\infty}\left(Q_{t}\right), v(t)=0 .
$$

Our goal is to pass to the limit in inequality (3.51). Indeed, by using the results of Proposition 3.4 and the weak lower semi-continuity of the energy function $E(t)$, we can easily pass to the limit in inequality (3.51) to obtain that $u$ satisfies the variational inequality (3.2). Moreover, since we also have the a priori regularity (see (3.49) )

$$
u \in C_{w}\left([0, T] ; H^{1}(\Omega)\right) \cap C_{w}^{1}\left([0, T] ; L_{2}(\Omega)\right),
$$


we may apply (3.2) with $v=0$ to obtain

$$
\int_{Q_{T}}|w|^{k} j\left(u_{t}\right) d Q_{T}<\infty
$$

Therefore, $u \in F(w)$ as desired.

\subsection{Proper proof of Theorem 1.6.}

Proof. Since $F(K)$ is compact, $F$ is upper semi-continuous (Lemma 3.6), $F(w)$ is convex, and the a priori bound holds in Lemma 2.1 (in the case $p>k+m$ the time $T$ may be finite), then by applying a standard truncation device for the mapping $F$ we are in a position to apply Kakutani's Theorem. Indeed, let $R$ be large enough so that for any $u \in \gamma F(u)$, where $0<\gamma<1$, we have

$$
|u|_{C\left([0, T] ; L_{q}(\Omega)\right)}<R \text {. }
$$

Indeed, $R$ can be determined by using the a priori bound Lemma 2.1 and the Sobolev embedding $H^{1}(\Omega) \hookrightarrow L_{q}(\Omega)$. We choose $K$ to be a ball of radius $R$ in $C\left([0, T] ; L_{q}(\Omega)\right)$ centered at the origin. Specifically, we set $K \equiv B_{C\left(L_{q}\right)}(0, R)$, where $C\left(L_{q}\right) \equiv C\left([0, T] ; L_{q}(\Omega)\right)$.

Next, we define the truncated mapping $F_{R}$ as follows:

$$
y_{R} \in F_{R}(w) \text { iff }\left\{\begin{array}{l}
y_{R}=y, y \in F(w) \cap B_{C\left(L_{q}\right)}(0, R), \\
\frac{R}{|y|_{C\left(L_{q}\right)}} y, y \in F(w),|y|_{C\left(L_{q}\right)}>R .
\end{array}\right.
$$

Thus, $F_{R}\left(C\left(L_{q}\right)\right) \subset K$ and $F_{R}$ satisfies all assumptions of Kakutani's Theorem (see 34, Theorem 9B, page 452). Therefore, $F_{R}$ has a fixed point, i.e., there exists $u \in C\left([0, T] ; L_{q}(\Omega)\right)$ such that $u \in F_{R}(u)$. At this end, we note that we have two possibilities. Either $u \in F(u)$ or else $u \in \gamma F(u)$, where $\gamma=\frac{R}{|y|_{C\left(L_{q}\right)}}<1$ for some $y \in F(u),|y|_{C\left(L_{q}\right)}>R$. However, the latter case cannot occur since if it did, then we must have $|u|_{C\left(L_{q}\right)}=R$. But this contradicts the a priori bound $|u|_{C\left(L_{q}\right)}<R$. Thus, we must have $u \in F(u)$ as desired.

Remark 3.7. In the special case when $k=0$ the argument is much simpler and the conclusions obtained are stronger than what has been stated in Proposition 3.4 Indeed, if $k=0$, then the strong monotonicity condition imposed on $\partial j$ allows us to prove the strong convergence: $u_{n t} \rightarrow u_{t}$ in $L_{m+1}\left(Q_{t}\right)$, where $u_{n}$ satisfies equation (3.7). This follows from (3.16) after setting $k=0$. Having obtained the strong convergence $u_{n t} \rightarrow u_{t}$ in $L_{m+1}\left(Q_{t}\right)$, we likewise obtain the strong convergence: $j\left(u_{n t}\right) \rightarrow j\left(u_{t}\right)$ in $L_{1}\left(Q_{t}\right)$. Based on the strong convergence of $j\left(u_{n t}\right)$ we can pass to the limit in equation (3.7) proving that $u=F(w)$, where $F(w)$ is defined by the variational equality and not inequality. In addition, the uniqueness of solutions is a direct consequence of monotonicity.

\section{Proof of Corollary 1.8}

4.1. Proof of the existence part. In order to establish the existence of weak solutions, it suffices to prove that generalized solutions satisfy the variational equality (1.7), under the additional restrictions imposed on the parameters $m, k$. 
Lemma 4.1. In addition to Assumption 1.1 and condition (1.4), assume the validity of condition (1.9). Then, every generalized solution to (1.1) satisfies the variational equality (1.7). Moreover, $\Delta u-u_{t t} \in L_{2}\left(Q_{t}\right)$.

Proof. We recall the restriction imposed on the parameters $k, m$ in (1.9). In this case, since $|u|^{k} \in L_{\frac{p^{*}}{k}}\left(Q_{t}\right), j(v) \in L_{\frac{2}{m+1}}\left(Q_{t}\right)$ for all functions $v \in L_{2}\left(Q_{t}\right)$, it follows that

$$
\int_{Q_{t}}|u|^{k} j(v) d t d x \leq C\left(\left|u_{0}\right|_{1, \Omega},\left|u_{1}\right|_{0, \Omega},|v|_{L_{2}\left(Q_{t}\right)}\right)
$$

and

$$
\int_{Q_{t}}|u|^{k} j\left(u_{t}\right) d t d x \leq C\left(\left|u_{0}\right|_{1, \Omega},\left|u_{1}\right|_{0, \Omega}\right) .
$$

Since we already know that the solution $u$ satisfies the variational inequality (1.5), then by applying the above bounds along with $|u|^{p} \in L_{2}(\Omega)$ implied by $p \leq \frac{p^{*}}{2}$ (which follows from (1.4) and (1.9)), we have

$$
\int_{Q_{t}}\left(u_{t t}-\Delta u\right) v d x d t \leq C\left(\left|u_{0}\right|_{1, \Omega},\left|u_{1}\right|_{0, \Omega},|v|_{L_{2}\left(Q_{t}\right)}\right),
$$

for all $v \in L_{2}\left(Q_{t}\right)$. This shows that $\square u \equiv u_{t t}-\Delta u$ is defined in the sense of distributions and satisfies

$$
\square u \in L_{2}\left(Q_{t}\right) .
$$

Moreover, by referring to classical arguments in the linear theory and keeping in mind (4.4), we also have the following energy identity which can be derived by a standard approximation argument

$$
\int_{0}^{t} \int_{\Omega}\left(u_{t t}-\Delta u\right) u_{t} d x d t=\frac{1}{2} \int_{\Omega}\left[\left|u_{t}(t)\right|^{2}+|\nabla u(t)|^{2}-\left|u_{t}(0)\right|^{2}-|\nabla u(0)|^{2}\right] d x .
$$

Therefore, in this case, the variational inequality (1.5) can be rewritten as

$$
\begin{array}{r}
\int_{0}^{t} \int_{\Omega}\left(\Delta u-u_{t t}\right) v d x d t+\frac{1}{2} \int_{\Omega}\left[u_{t}^{2}(t)+|\nabla u(t)|^{2}\right] d x \\
+\int_{0}^{t} \int_{\Omega}|u|^{k}\left[j\left(u_{t}\right)-j(v)\right] d x d t \\
\leq \int_{0}^{t} \int_{\Omega}|u|^{p-1} u\left(u_{t}-v\right) d x d t+\frac{1}{2} \int_{\Omega}\left[u_{1}^{2}+\left|\nabla u_{0}\right|^{2}\right] d x,
\end{array}
$$

for all test functions $v \in L_{2}\left(Q_{t}\right)$. Here, we note that (4.6) is first obtained with test functions satisfying the requirements in (1.5), and then by density, (4.6) is extended to all test functions $v \in L_{2}\left(Q_{t}\right)$.

Now, since $u_{t} \in L_{2}\left(Q_{t}\right)$ we are in a position to set $v$ in (4.6) as follows:

$$
v \equiv u_{t}+\lambda \psi, \psi \in L_{2}\left(Q_{t}\right), \lambda>0 .
$$

By using the energy identity (4.5) and after simplification, for all test functions $\psi \in L_{2}\left(Q_{t}\right)$ we obtain

$$
\begin{gathered}
\int_{0}^{t} \int_{\Omega}\left(\Delta u-u_{t t}\right) \psi d x d t+\frac{1}{\lambda} \int_{0}^{t} \int_{\Omega}|u|^{k}\left[j\left(u_{t}\right)-j\left(u_{t}+\lambda \psi\right)\right] d x d t \\
\leq \int_{0}^{t} \int_{\Omega}|u|^{p-1} u \psi d x d t .
\end{gathered}
$$


Our final step is to establish the following limit:

$$
\frac{1}{\lambda} \int_{0}^{t} \int_{\Omega}|u|^{k}\left[j\left(u_{t}\right)-j\left(u_{t}+\lambda \psi\right)\right] d x d t \rightarrow-\int_{0}^{t} \int_{\Omega}|u|^{k} \partial j\left(u_{t}\right) \psi d x d t
$$

as $\lambda \rightarrow 0^{+}$.

To establish (4.8), we first note that

$$
\frac{1}{\lambda}\left[j\left(u_{t}\right)-j\left(u_{t}+\lambda \psi\right)\right] \rightarrow-\partial j\left(u_{t}\right) \psi \text { almost everywhere in } Q_{t}, \text { as } \lambda \rightarrow 0^{+} .
$$

Hence, as $\lambda \rightarrow 0^{+}$,

$$
\frac{1}{\lambda}|u|^{k}\left[j\left(u_{t}\right)-j\left(u_{t}+\lambda \psi\right)\right] \rightarrow|u|^{k} \partial j\left(u_{t}\right) \psi \text { almost everywhere in } Q_{t} .
$$

On the other hand by mean value theorem and the growth condition on $\partial j$, we have the estimate

$$
\begin{aligned}
& \frac{1}{\lambda}\left[j\left(u_{t}\right)-j\left(u_{t}+\lambda \psi\right)\right]=-\psi \int_{0}^{1} \partial j\left(u_{t}+(1-s) \lambda \psi\right) d s \\
& \leq C\left[\left|u_{t}\right|^{m}+|\psi|^{m}+1\right]|\psi| .
\end{aligned}
$$

By recalling the restrictions on the parameters, we deduce that

$$
\int_{Q_{t}}|u|^{k}\left|u_{t}\right|^{m}|\psi| d x d t<\infty, \int_{Q_{t}}|u|^{k}\left[|\psi|^{m}+1\right]|\psi| d x d t<\infty,
$$

for every test function $\psi \in L_{2}\left(Q_{t}\right)$.

The final conclusion in (4.8) follows immediately from the Lebesgue Dominated Convergence Theorem.

Finally, by using (4.8) and passing to the limit as $\lambda \rightarrow 0^{+}$in variational inequality, 4.7] leads to the inequality

$$
\int_{Q_{t}}\left(\Delta u-u_{t t}\right) \psi d x d t-\int_{Q_{t}}|u|^{k} \partial j\left(u_{t}\right) \psi d x d t \leq-\int_{Q_{t}}|u|^{p-1} u \psi d x d t,
$$

for all test functions $\psi \in L_{2}\left(Q_{t}\right)$. Since $\psi$ is arbitrary we obtain the equality

$$
\int_{Q_{t}}\left(\Delta u-u_{t t}\right) \psi d x d t-\int_{Q_{t}}|u|^{k} \partial j\left(u_{t}\right) \psi d x d t=-\int_{Q_{t}}|u|^{p-1} u \psi d x d t
$$

for all test functions $\psi \in L_{2}\left(Q_{t}\right)$.

This implies the variational equality in (1.7), completing the proof of the existence of weak solutions as claimed in lemma 4.1

4.2. Proof of the uniqueness statement in Corollary 1.8. In order to establish the uniqueness statement in Corollary 1.8 we prove the following lemma.

Lemma 4.2. In addition to Assumption 1.1 and conditions (1.4), (1.9), assume $k, p \geq 1$. Then, the weak solution of (1.1) (established in Lemma 4.1) is unique. More precisely, if $u, v \in C_{w}\left([0, T], H_{0}^{1}(\Omega)\right)$ and $u^{\prime}, v^{\prime} \in C_{w}\left([0, T], L^{2}(\Omega)\right)$ such that $u$ and $v$ are weak solutions to the initial-boundary value problem (1.1) in the sense of Definition [1.5, then $u=v$.

Proof. Let $w=u-v$. Let $L>0$ be such that

$$
|u(t)|_{1, \Omega},|v(t)|_{1, \Omega},\left|u^{\prime}(t)\right|_{0, \Omega},\left|v^{\prime}(t)\right|_{0, \Omega} \leq L,
$$

for all $t \in[0, T]$. 
First note that $w$ verifies

$$
\begin{gathered}
w_{t t}-\Delta w+|u|^{k} \partial j\left(u_{t}\right)-|v|^{k} \partial j\left(v_{t}\right)=f(u)-f(v) \quad \text { in } \Omega \times(0, T), \\
w(x, 0)=0, \quad w_{t}(x, 0)=0 \quad \text { in } \Omega, \\
w(x, t)=0 \text { on } \Gamma \times(0, T),
\end{gathered}
$$

where $f(u)=|u|^{p-1} u$. In particular, $w$ satisfies the following energy inequality:

$$
\begin{aligned}
& \frac{1}{2}\left(\left\|w^{\prime}(t)\right\|_{L_{2}(\Omega)}^{2}+|w(t)|_{1, \Omega}^{2}\right) \\
& \quad+\int_{0}^{t}\left\langle|u(\tau)|^{k}\left[\partial j\left(u^{\prime}\right)-\partial j\left(v^{\prime}\right)\right], w^{\prime}(\tau)\right\rangle_{L_{2}(\Omega)} d \tau \\
& \leq-\int_{0}^{t}\left\langle\left[|u(\tau)|^{k}-|v(\tau)|^{k}\right] \partial j\left(v^{\prime}\right), w^{\prime}(\tau)\right\rangle_{L_{2}(\Omega)} d \tau \\
& \quad+\int_{0}^{t}\left\langle f(u(\tau))-f(v(\tau)), w^{\prime}(\tau)\right\rangle_{L_{2}(\Omega)} d \tau .
\end{aligned}
$$

In view of the strict monotonicity in Assumption 1.1, the left-hand side of (4.13) is nonnegative. By using the continuity assumption in Assumption 1.1 and the elementary inequality

$$
\left.|| a\right|^{k}-|b|^{k}|\leq C| a-b \mid\left(|a|^{k-1}+|b|^{k-1}\right),
$$

for some constant $C>0$, all $k \geq 1$, and all $a, b \in \mathbb{R}$, we have

$$
\begin{aligned}
-\left\langle\left[|u(\tau)|^{k}\right.\right. & \left.\left.-|v(\tau)|^{k}\right]\left|\partial j\left(v^{\prime}\right)\right|, w^{\prime}(\tau)\right\rangle_{L_{2}(\Omega)} \\
& \leq C\left\langle\left|v^{\prime}\right|^{m}|u-v|\left[|u|^{k-1}+|v|^{k-1}\right],\left|w^{\prime}\right|\right\rangle_{L_{2}(\Omega)} .
\end{aligned}
$$

For space dimensions $n \geq 3$, we recall the assumption $\frac{k}{p^{*}}+\frac{m}{2} \leq \frac{1}{2}$, and choose

$$
\alpha=\frac{2}{m}, \beta=\frac{2 n}{n-2}, \gamma=\frac{2 n}{(n-2)(k-1)}, \delta=\frac{2 n}{2(n+k)-n(m+k)} .
$$

In this case, it is easy to see that $\alpha, \beta, \gamma, \delta$ are Hölder's conjugate exponents, and in particular $1<\delta \leq 2$. Both terms on the left-hand side of (4.14) are estimated in the same way. By using the generalized Hölder inequality and the Sobolev imbedding theorem, we have

$$
\begin{aligned}
& \left\langle\left|v^{\prime}(\tau)\right|^{m}|u(\tau)-v(\tau)|\left[|u(\tau)|^{k-1}+|v(\tau)|^{k-1}\right],\left|w^{\prime}(\tau)\right|\right\rangle_{L_{2}(\Omega)} \\
& \quad \leq\left\|v_{2}^{\prime}(\tau)\right\|_{L_{2}(\Omega)}^{m}\|w(\tau)\|_{L_{p^{*}}(\Omega)}\left[\|u(\tau)\|_{L_{p^{*}}(\Omega)}^{k-1}+\|v(\tau)\|_{L_{p^{*}}(\Omega)}^{k-1}\right]\left\|w^{\prime}(\tau)\right\|_{L_{\delta}(\Omega)} \\
& \quad \leq C\left\|v_{2}^{\prime}(\tau)\right\|_{L_{2}(\Omega)}^{m}|w(\tau)|_{1, \Omega}\left[|u(\tau)|_{1, \Omega}^{k-1}+|v(\tau)|_{1, \Omega}^{k-1}\right]\left\|w^{\prime}(\tau)\right\|_{L_{2}(\Omega)} \\
& \quad \leq C L^{m+k-1}|w(\tau)|_{1, \Omega}\left\|w^{\prime}(\tau)\right\|_{L_{2}(\Omega)} \\
& \quad \leq C\left[\left\|w^{\prime}(\tau)\right\|_{L_{2}(\Omega)}^{2}+|w(\tau)|_{1, \Omega}^{2}\right]
\end{aligned}
$$

for all $\tau \in[0, T]$. 
For space dimensions $n=1,2$ we only impose the assumption $0<m<1$. Here, we choose $\beta>1$ large enough so that the following:

$$
\alpha=\frac{2}{m}, \beta=\gamma, \delta=\frac{2 \beta}{(2-m) \beta-4}
$$

are Hölder's conjugate exponents, with $1<\delta \leq 2$. Thus, for $n=1,2$, 4.15) is still valid.

Similarly, by noting that $1 \leq p \leq \frac{p^{*}}{2}=\frac{n}{n-2}$ which implies $\frac{2(p-1) p^{*}}{p^{*}-2}=n(p-1) \leq$ $p^{*}$, we then have

$$
\begin{aligned}
\langle f(u(\tau) & )-f(v(\tau)), w^{\prime}(\tau)\right\rangle_{L_{2}(\Omega)} \\
= & \left\langle|u(\tau)|^{p-1} u(\tau)-|v(\tau)|^{p-1} v(\tau), w^{\prime}(\tau)\right\rangle_{L_{2}(\Omega)} \\
\leq & C\left\langle|u(\tau)-v(\tau)|\left[|u(\tau)|^{p-1}+|v(\tau)|^{p-1}\right],\left|w^{\prime}(\tau)\right|\right\rangle_{L_{2}(\Omega)} \\
\leq & C\|w(\tau)\|_{L_{p^{*}}(\Omega)}\left\|w^{\prime}(\tau)\right\|_{L_{2}(\Omega)}\left[\|u(\tau)\|_{L_{n(p-1)}(\Omega)}^{p-1}+\|v(\tau)\|_{L_{n(p-1)}(\Omega)}^{p-1}\right] \\
\leq & C|w(\tau)|_{1, \Omega}\left\|w^{\prime}(\tau)\right\|_{L_{2}(\Omega)}\left[|u(\tau)|_{1, \Omega}^{p-1}+|v(\tau)|_{1, \Omega}^{p-1}\right] \\
\quad \leq & C\left[\left\|w^{\prime}(\tau)\right\|_{L_{2}(\Omega)}^{2}+|w(\tau)|_{1, \Omega}^{2}\right] .
\end{aligned}
$$

We remark here that the estimates in (4.16) are also valid for the space dimensions $n=1,2$, by a similar argument. Therefore, it follows from (4.13)-(4.16) that

$$
\left\|w^{\prime}(t)\right\|_{L_{2}(\Omega)}^{2}+|w(t)|_{1, \Omega}^{2} \leq C \int_{0}^{t}\left[\left\|w^{\prime}(\tau)\right\|_{L_{2}(\Omega)}^{2}+|w(\tau)|_{1, \Omega}^{2}\right] d \tau
$$

for $t \in[0, T]$. Hence, by Gronwall's inequality

$$
\left\|w^{\prime}(t)\right\|_{L_{2}(\Omega)}^{2}+|w(t)|_{1, \Omega}^{2}=0, \text { for } t \in[0, T] .
$$

Finally, we note that the same estimate as the one above shows that solutions are continuously dependent with respect to the initial data in the $H^{1} \times L_{2}$ norms. This completes the proof of the uniqueness statement in Corollary 1.8 .

\section{Proof of Theorem 1.9}

As for the existence part of Theorem 1.9 the arguments are routine. This is due to the fact that the damping is linear in $u_{t}$, and there are no technical difficulties in passing to the limit in the nonlinear damping term. The main challenge is proof of uniqueness. Therefore, it suffices to prove uniqueness of solutions within the context of the assumptions imposed in Theorem 1.9, For this, the argument is very different than before, and it is based on a certain change of variables which reduces the problem to a parabolic-like system. In fact, while we are able to establish the uniqueness of solutions in this case, as stated in Lemma [5.1, the same cannot be said about the continuous dependence with respect to the initial conditions in the finite energy norm.

Lemma 5.1. In addition to the assumptions stated in Theorem 1.9, assume that $p \geq 1$. Then, the weak solution of (1.3) (with $m=1$ ) is unique. 
Proof. Since $m=1$ we consider

$$
u_{t t}+|u|^{k} u_{t}-\Delta u=|u|^{p-1} u .
$$

Let $u$ be a finite energy weak solution to (5.1). Let $g(s)$ be the antiderivative of $|s|^{k}$, so that $g^{\prime}(s)=|s|^{k}$.

Let $z \equiv u_{t}+g(u)$. Since $u \in C_{w}\left([0, T], H_{0}^{1}(\Omega)\right) \cap C_{w}^{1}\left([0, T], L_{2}(\Omega)\right)$ and $|g(s)| \leq$ $|s|^{k+1}$ where $k+1 \leq \frac{1}{2} p^{*}$, we then have

$$
z \in C_{w}^{1}\left([0, T], L_{2}(\Omega)\right) .
$$

We also note that

$$
u_{t t}+|u|^{k} u_{t}=z_{t} .
$$

Thus, $u \in C_{w}\left([0, T] ; H_{0}^{1}(\Omega)\right)$ and $z \in C_{w}\left([0, T], L_{2}(\Omega)\right)$ are solutions of the following system of equations:

$$
\begin{aligned}
u_{t}+g(u) & =z, \\
z_{t}-\Delta u & =|u|^{p-1} u, u=0 \text { on } \partial \Omega .
\end{aligned}
$$

Since $g$ is monotone, by a standard argument in abstract ordinary differential equations we can solve the first equation (5.2) for $u$ to obtain

$$
u=R\left(z, u_{0}\right) .
$$

Moreover, we have the following standard estimate:

$$
|u(t)|_{0, \Omega}^{2}+\int_{0}^{t} \int_{\Omega}|u(s)|^{k+2} d x d s \leq c_{T}\left(\left|u_{0}\right|_{0, \Omega}^{2}+\int_{0}^{T}|z|_{0, \Omega}^{2} d t\right),
$$

for all $t \in[0, T]$.

Now Let $u$ and $v$ be two solutions of (5.1) with the same initial data. Let $L>0$ be such that $|u(t)|_{1, \Omega}$ and $|v(t)|_{1, \Omega} \leq L$ for all $t \in[0, T]$. We shall show that $u \equiv v$.

At this end, we let $U(t)=u(t)-v(t), Z(t)=z_{1}(t)-z_{2}(t)$, where $z_{1}(t)=$ $u_{t}(t)+g(u(t)), z_{2}(t)=v_{t}(t)+g(v(t))$. Hence,

$$
U_{t}+g(u)-g(v)=Z
$$

and

$$
Z_{t}-\Delta U=|u|^{p-1} u-|v|^{p-1} v .
$$

By exploiting the monotonicity of $g$, it then follows from (5.5) that

$$
|U(t)|_{0, \Omega}^{2} \leq|U(0)|_{0, \Omega}^{2}+2 \int_{0}^{t} \int_{\Omega} U(s) Z(s) d x d s .
$$

By recalling the equation for $z$ in (5.2), we infer that

$$
A^{-1} z_{t}+u=A^{-1}\left(|u|^{p-1} u\right),
$$

where $A^{-1}$ denotes the inverse of the operator $-\triangle$ with the zero boundary conditions. In particular, (5.6) yields

$$
A^{-1} Z_{t}+U=A^{-1}\left[|u|^{p-1} u-|v|^{p-1} v\right] .
$$


By taking the $L^{2}$-inner product with $Z$ and integrating in time, we obtain

$$
\begin{aligned}
& |Z(t)|_{-1, \Omega}^{2}+\int_{0}^{t}\left(R\left(z_{1}, u_{0}\right)-R\left(z_{2}, u_{0}\right), Z\right)_{\Omega} \\
& \leq\left.\int_{0}^{t}|Z(s)|_{-1, \Omega}|| R\left(z_{1}, u_{0}\right)\right|^{p-1} R\left(z_{1}, u_{0}\right)-\left.\left|R\left(z_{2}, u_{0}\right)\right|^{p-1} R\left(z_{2}, u_{0}\right)\right|_{-1, \Omega} d t .
\end{aligned}
$$

By recalling (5.7), we have

$$
2 \int_{0}^{t}\left(R\left(z_{1}, u_{0}\right)-R\left(z_{2}, u_{0}\right), Z\right)_{\Omega} \geq|U(t)|_{0, \Omega}^{2}-|U(0)|_{0, \Omega}^{2}=|U(t)|_{0, \Omega}^{2} .
$$

However, by using duality

$$
|u|_{-1, \Omega}=\sup _{\phi \in H_{0}^{1}(\Omega)} \frac{(u, \phi)_{\Omega}}{|\phi|_{1, \Omega}}
$$

along with the elementary inequality

$$
\left.|| a\right|^{p-1} a-|b|^{p-1} b|\leq C| a-b \mid\left[|a|^{p-1}+|b|^{p-1}\right]
$$

we obtain

$$
\begin{aligned}
\mid Z(t) & \left.\right|_{-1, \Omega} ^{2}+|U(t)|_{0, \Omega}^{2} \\
& \leq\left.\int_{0}^{t}|Z(s)|_{-1, \Omega}|| R\left(z_{1}, u_{0}\right)\right|^{p-1} R\left(z_{1}, u_{0}\right)-\left.\left|R\left(z_{2}, u_{0}\right)\right|^{p-1} R\left(z_{2}, u_{0}\right)\right|_{-1, \Omega} d s \\
& \leq C \int_{0}^{t}|Z(s)|_{-1, \Omega}|U(s)|_{0, \Omega}\left[\left\|R\left(z_{1}, u_{0}\right)\right\|_{\frac{L^{2(p-1) p^{*}}}{p^{*}-2}}^{p-1}+\left\|R\left(z_{2}, u_{0}\right)\right\|_{\frac{2(p-1) p^{*}}{p^{*}-2}}^{p-1}\right] d s .
\end{aligned}
$$

Since $p \leq \frac{p^{*}}{2}$, then $\frac{2 p^{*}(p-1)}{p^{*}-2} \leq p^{*}$, and therefore $\left\|R\left(z_{i}, u_{0}\right)\right\|_{\frac{2(p-1) p^{*}}{p^{*}-2}}^{p-1} \leq C(L)$, for $i=1,2$. Hence, it follows from (5.10) that

$$
|Z(t)|_{-1, \Omega}^{2}+|U(t)|_{0, \Omega}^{2} \leq C \int_{0}^{t}\left[|Z(t)|_{-1, \Omega}^{2}+|U(t)|_{0, \Omega}^{2}\right] d s
$$

which completes the proof.

\section{Proof of Theorem 1.13-Blow-up of SOlutions}

Throughout this section, we assume that $p>k+m$ and $u$ is a weak solution of (1.1) in the sense of Definition 1.5 In particular, $u$ verifies the following energy identity:

$$
\begin{aligned}
E(t):=\frac{1}{2}\left(\left\|u^{\prime}(t)\right\|_{L^{2}(\Omega)}^{2}\right. & \left.+\left\|A^{1 / 2} u(t)\right\|_{L^{2}(\Omega)}^{2}\right)-\frac{1}{p+1}\|u(t)\|_{L^{p+1}(\Omega)}^{p+1} \\
& +\int_{0}^{t} \int_{\Omega}|u(\tau)|^{k} \partial j\left(u^{\prime}(\tau)\right) u^{\prime}(\tau) d x d \tau=E(0) .
\end{aligned}
$$

Indeed, the above energy equality follows from known arguments, due to the fact that $u_{t t}-\Delta u \in L_{2}\left(Q_{T}\right)$ (see [12]).

As we mentioned earlier in the Introduction, the first global nonexistence result for the degenerate case $k>0$ was pointed out in a Remark below Theorem 4 in [16] where the authors of [16] explicitly identified the region $m+k<p$ as the region for 
global nonexistence. Also, the proof of Theorem 1.13 below draws substantially on the ideas introduced in [6], including the choice of the special Liapunov's function.

We now proceed to prove Theorem 1.13.

Proof. As in [6], Let

$$
\begin{gathered}
F(t)=\|u(t)\|_{L^{2}(\Omega)}^{2} \\
H(t)=-\frac{1}{2}\left(\left\|u^{\prime}(t)\right\|_{L^{2}(\Omega)}^{2}+\left\|A^{1 / 2} u(t)\right\|_{L^{2}(\Omega)}^{2}\right)+\frac{1}{p+1}\|u(t)\|_{L^{p+1}(\Omega)}^{p+1} .
\end{gathered}
$$

Note that the assumption that $E(0)<0$ is equivalent to $H(0)>0$.

First, (6.1) and the strict monotonicity in Assumption 1.1 yields that

$$
H^{\prime}(t)=\int_{\Omega}|u(t)|^{k} \partial j\left(u^{\prime}(t)\right) u^{\prime}(t) d x \geq c \int_{\Omega}|u(t)|^{k}\left|u^{\prime}(t)\right|^{m+1} d x \geq 0 .
$$

Therefore,

$$
0<H(0) \leq H(t) \leq \frac{1}{p+1}\|u(t)\|_{L^{p+1}(\Omega)}^{p+1},
$$

for $0 \leq t<T$.

Let $\alpha=\min \left\{\frac{p-(k+m)}{m(p+1)}, \frac{p-1}{2(p+1)}\right\}$. In particular, $0<\alpha<\frac{1}{2}$. Let $K$ and $L$ be the constant given by

$$
K=2 \frac{c_{0}}{c^{m /(m+1)}}|\Omega|^{\frac{p-k-m}{(m+1)(p+1)}} \quad \text { and } \quad L=\frac{(p+1)^{\frac{k+m+1}{m(p+1)}}}{(p-1)^{\frac{1}{m}}} H(0)^{\alpha-\frac{p-k-m}{m(p+1)}},
$$

where $c, c_{0}$ are the constants that appear in Assumption 1.1. Let $0<\epsilon<1$ be small enough so that

$$
1-\alpha-\epsilon K^{1+\frac{1}{m}} L \geq 0
$$

Later, we may need to adjust $\epsilon$ again.

In the remainder of the proof, most generic constants will be denoted by $C$, $C_{0}, \ldots$. They may depend on various parameters, but they are totally independent from $\epsilon$ and the initial data, and they may change from line to line.

First, we note that 6.6 implies

$$
H(0) \geq C \epsilon^{\theta}
$$

where $\theta=\frac{m(p+1)}{p-(k+m)-\alpha m(p+1)}>0$.

As in 6], we let

$$
y(t)=H(t)^{1-\alpha}+\epsilon F^{\prime}(t) .
$$

It follows from Definition 1.5 that $u^{\prime \prime} \in C_{w}\left([0, T), H^{-1}(\Omega)\right)$ and consequently, $F^{\prime \prime}(t)$ exists for $t \in[0, T)$ with

$$
\begin{aligned}
F^{\prime \prime}(t) & =2\left(\left\|u^{\prime}(t)\right\|_{L^{2}(\Omega)}^{2}-\left\|A^{1 / 2} u(t)\right\|_{L^{2}(\Omega)}^{2}+\|u(t)\|_{L^{p+1}(\Omega)}^{p+1}\right) \\
& -2 \int_{\Omega}|u(t)|^{k} u(t) \partial j\left(u^{\prime}(t)\right) d x .
\end{aligned}
$$


Therefore, (6.8)- 6.9) yield

$$
\begin{aligned}
y^{\prime}(t) & =(1-\alpha) H(t)^{-\alpha} H^{\prime}(t)+4 \epsilon\left\|u^{\prime}(t)\right\|_{L^{2}(\Omega)}^{2}+4 \epsilon H(t) \\
& +2 \epsilon \frac{p-1}{p+1}\|u(t)\|_{L^{p+1}(\Omega)}^{p+1}-2 \epsilon \int_{\Omega}|u(t)|^{k} u(t) \partial j\left(u^{\prime}(t)\right) d x .
\end{aligned}
$$

Since $p>k+m$, then by invoking the continuity of $\partial j$ in Assumption 1.1 and by using Hölder's inequality one has

$$
\begin{aligned}
& \left.\left.\left|\int_{\Omega}\right| u(t)\right|^{k} u(t) \partial j\left(u^{\prime}(t)\right) d x\left|\leq c_{0} \int_{\Omega}\right| u(t)\right|^{k+1-\frac{k m}{m+1}}|u(t)|^{\frac{k m}{m+1}}\left|u^{\prime}(t)\right|^{m} d x \\
& \quad \leq c_{0}\left(\int_{\Omega}|u(t)|^{k}\left|u^{\prime}(t)\right|^{m+1} d x\right)^{\frac{m}{m+1}}\left(\int_{\Omega}|u(t)|^{k+m+1} d x\right)^{\frac{1}{m+1}} \\
& \quad \leq \frac{1}{2} K H^{\prime}(t)^{\frac{m}{m+1}}\|u(t)\|_{L^{p+1}(\Omega)}^{\frac{k+m+1}{m+1}},
\end{aligned}
$$

where $K$ is as given in (6.5). However, Young's inequality and (6.11) yield

$$
\left.\left|\int_{\Omega}\right| u(t)\right|^{k} u(t) g\left(u^{\prime}(t)\right) d x \mid \leq \frac{1}{2} K\left[\frac{1}{\delta} H^{\prime}(t)+\delta^{m}\|u(t)\|_{L^{p+1}(\Omega)}^{k+m+1}\right],
$$

where $\delta>0$ is to be chosen later. Therefore, it follows from (6.10) and 6.12 that

$$
\begin{aligned}
y^{\prime}(t) \geq & {\left[(1-\alpha) H(t)^{-\alpha}-K \frac{\epsilon}{\delta}\right] H^{\prime}(t)+4 \epsilon\left\|u^{\prime}(t)\right\|_{L^{2}(\Omega)}^{2} } \\
& +4 \epsilon H(t)+2 \epsilon \frac{p-1}{p+1}\|u(t)\|_{L^{p+1}(\Omega)}^{p+1}-K \epsilon \delta^{m}\|u(t)\|_{L^{p+1}(\Omega)}^{k+m+1} .
\end{aligned}
$$

By choosing $\delta=\left[\frac{p-1}{(p+1) K}\|u(t)\|_{L^{p+1}(\Omega)}^{p-k-m}\right]^{\frac{1}{m}}$, then

$$
\epsilon \frac{p-1}{p+1}\|u(t)\|_{L^{p+1}(\Omega)}^{p+1}-K \epsilon \delta^{m}\|u(t)\|_{L^{p+1}(\Omega)}^{k+m+1}=0 .
$$

Therefore, we have

$$
\begin{aligned}
y^{\prime}(t) & \geq\left[(1-\alpha) H(t)^{-\alpha}-K \frac{\epsilon}{\delta}\right] H^{\prime}(t)+4 \epsilon\left\|u^{\prime}(t)\right\|_{L^{2}(\Omega)}^{2} \\
& +4 \epsilon H(t)+\epsilon \frac{p-1}{p+1}\|u(t)\|_{L^{p+1}(\Omega)}^{p+1} .
\end{aligned}
$$

Since $H(t) \leq \frac{1}{p+1}\|u(t)\|_{L^{p+1}(\Omega)}^{p+1}$, then

$$
\begin{aligned}
& (1-\alpha) H(t)^{-\alpha}-K \frac{\epsilon}{\delta}=H^{-\alpha}(t)\left[1-\alpha-K \frac{\epsilon}{\delta} H(t)^{\alpha}\right] \\
& \geq H(t)^{-\alpha}\left[1-\alpha-\epsilon K^{1+\frac{1}{m}} \frac{(p+1)^{\frac{1}{m}-\alpha}}{(p-1)^{\frac{1}{m}}}\|u(t)\|_{L^{p+1}(\Omega)}^{\frac{k+m-p+\alpha m(p+1)}{m}}\right] .
\end{aligned}
$$

Furthermore, since $\|u(t)\|_{L^{p+1}(\Omega)} \geq[(p+1) H(0)]^{\frac{1}{p+1}}>0$ and $\alpha$ was chosen so that $k+m-p+\alpha m(p+1) \leq 0$, it then follows from (6.15) that

$$
\begin{aligned}
& (1-\alpha) H(t)^{-\alpha}-K \frac{\epsilon}{\delta} \\
& \geq H(t)^{-\alpha}\left[1-\alpha-\epsilon K^{1+\frac{1}{m}} \frac{(p+1)^{\frac{k+m+1}{m(p+1)}}}{(p-1)^{\frac{1}{m}}} H(0)^{\alpha-\frac{p-k-m}{m(p+1)}}\right] \\
& \quad \equiv H(t)^{-\alpha}\left[1-\alpha-\epsilon K^{1+\frac{1}{m}} L\right] \geq 0,
\end{aligned}
$$


by our choice of $\epsilon$ in (6.6). Therefore, (6.14) and (6.16) yield

$$
y^{\prime}(t) \geq \epsilon C\left[H(t)+\left\|u^{\prime}(t)\right\|_{L^{2}(\Omega)}^{2}+\|u(t)\|_{L^{p+1}(\Omega)}^{p+1}\right],
$$

for $t \in[0, T)$ and where $C>0$ is a constant that does not depend on $\epsilon$. In particular (6.17) shows that $y(t)$ is increasing on $[0, T)$, with

$$
y(t)=H(t)^{1-\alpha}+\epsilon F^{\prime}(t) \geq H(0)^{1-\alpha}+\epsilon F^{\prime}(0) .
$$

If $F^{\prime}(0) \geq 0$, then no further condition on $\epsilon$ is needed. However, if $F^{\prime}(0)<0$, then we further adjust $\epsilon$ so that $0<\epsilon \leq-\frac{H(0)^{1-\alpha}}{2 F^{\prime}(0)}$. In any case, one has $y(t)>0$, for $t \in[0, T)$.

Finally, we show that $y(t)$ satisfies the differential inequality

$$
y^{\prime}(t) \geq \epsilon^{1+\sigma} C_{0} y(t)^{\frac{1}{1-\alpha}}, \quad 0 \leq t<T,
$$

where $C_{0}$ is some positive constant and $\sigma=\theta\left(1-\frac{2}{(1-2 \alpha)(p+1)}\right) \geq 0$.

If $F^{\prime}(t) \leq 0$ for some $t \in[0, T)$, then for such values of $t$ we have

$$
y(t)^{\frac{1}{1-\alpha}}=\left[H(t)^{1-\alpha}+\epsilon F^{\prime}(t)\right]^{\frac{1}{1-\alpha}} \leq H(t) .
$$

Thus, (6.20) and (6.17) show that (6.19) is valid for all $t \in[0, T)$ for which $F^{\prime}(t) \leq 0$. If $t \in[0, T)$ is such that $F^{\prime}(t)>0$, then (6.19) will be valid, if for such values of $t \in[0, T)$ the following inequality holds:

$$
H(t)+\|u(t)\|_{L^{p+1}(\Omega)}^{p+1}+\left\|u^{\prime}(t)\right\|_{L^{2}(\Omega)}^{2} \geq \epsilon^{\sigma} C\left[H(t)^{1-\alpha}+\epsilon F^{\prime}(t)\right]^{\frac{1}{1-\alpha}} .
$$

So, assume that $F^{\prime}(t)>0$, and let $\beta=\frac{1}{1-\alpha}$. Since $1<\beta<2$ and $0<\epsilon<1$, then by convexity

$$
\left[H(t)^{1-\alpha}+\epsilon F^{\prime}(t)\right]^{\beta} \leq 2^{\beta-1}\left[H(t)+F^{\prime}(t)^{\beta}\right] .
$$

However, since $p \geq 1$, we have

$$
\begin{aligned}
F^{\prime}(t)^{\beta} & =\left(2 \int_{\Omega} u(t) u^{\prime}(t) d x\right)^{\beta} \leq C\left(\|u(t)\|_{L^{2}(\Omega)}\left\|u^{\prime}(t)\right\|_{L^{2}(\Omega)}\right)^{\beta} \\
& \leq C\|u(t)\|_{L^{p+1}(\Omega)}^{\beta}\left\|u^{\prime}(t)\right\|_{L^{2}(\Omega)}^{\beta} .
\end{aligned}
$$

Since $\frac{2}{\beta}=2(1-\alpha)>1$, then by using Young's inequality, we obtain

$$
F^{\prime}(t)^{\beta} \leq C\left(\left\|u^{\prime}(t)\right\|_{L^{2}(\Omega)}^{2}+\|u(t)\|_{L^{p+1}(\Omega)}^{\frac{2 \beta}{2-\beta}}\right) .
$$

Now, by recalling $\|u(t)\|_{L^{p+1}(\Omega)}^{p+1}>(p+1) H(0)>0$ and by noting that $\alpha \leq \frac{p-1}{2(p+1)}$ is equivalent to $\frac{2 \beta}{2-\beta}:=\frac{2}{1-2 \alpha} \leq p+1$, there exists a constant $C_{1}>0$ that is independent of $\epsilon$ and the initial data, such that

$$
\|u(t)\|_{L^{p+1}(\Omega)}^{\frac{2 \beta}{2-\beta}} \leq C_{1} H(0)^{\frac{2}{(1-2 \alpha)(p+1)}}-1\|u(t)\|_{L^{p+1}(\Omega)}^{p+1} .
$$

Since $\frac{2}{(1-2 \alpha)(p+1)}-1 \leq 0$, it then follows from (6.7) that

$$
\|u(t)\|_{L^{p+1}(\Omega)}^{\frac{2 \beta}{2-\beta}} \leq C \epsilon^{-\sigma}\|u(t)\|_{L^{p+1}(\Omega)}^{p+1},
$$

where

$$
\sigma=\theta\left(1-\frac{2}{(1-2 \alpha)(p+1)}\right)=\frac{m}{p-(k+m)-\alpha m(p+1)}\left(p+1-\frac{2}{(1-2 \alpha)}\right) \geq 0 .
$$


Thus, it follows from (6.26) and (6.24) that

$$
F^{\prime}(t)^{\frac{1}{1-\alpha}} \leq C \epsilon^{-\sigma}\left(\left\|u^{\prime}(t)\right\|_{L^{2}(\Omega)}^{2}+\|u(t)\|_{L^{p+1}(\Omega)}^{p+1}\right) .
$$

By combining (6.22) and (6.27), then (6.21) follows. Consequently (6.19) holds, and therefore, $y(t)=H(t)^{1-\alpha}+\epsilon F^{\prime}(t)$ blows up in finite time $T$, where

$$
T<C \epsilon^{-1-\sigma} y(0)^{-\frac{\alpha}{1-\alpha}} \text {. }
$$

Remark 6.1. If $F^{\prime}(0) \geq 0$, then (6.28) yields the following upper bound for the life span of the solution:

$$
T<C \epsilon^{-1-\sigma}\left[H(0)^{1-\alpha}+\epsilon F^{\prime}(0)\right]^{-\frac{\alpha}{1-\alpha}} \leq C \epsilon^{-1-\sigma} H(0)^{-\alpha} .
$$

However, if $F^{\prime}(0)<0$, then (6.29) is still valid, since we have chosen $\epsilon$ in the proof so that $0<\epsilon \leq-\frac{H(0)^{1-\alpha}}{2 F^{\prime}(0)}$. Now, if the initial data is sufficiently small, then in view of (6.7), $\epsilon \sim H(0)^{\frac{1}{\theta}}$, and therefore we have

$$
T<C H(0)^{-\alpha-\frac{1}{\theta}-\frac{\sigma}{\theta}}=C H(0)^{-\frac{p-(k+m)}{m(p+1)}-\left(1-\frac{2}{(1-2 \alpha)(p+1)}\right)} .
$$

\section{Proof of Theorem 1.10}

This section is devoted to addressing the issue of propagation of regularity. More specifically, if we assume that the initial data enjoy more regularity, then we can prove that the corresponding solution is more regular as well. In this case, the concept of a solution is classical in the sense that each term in the differential equation has point-wise meaning almost everywhere. The key to this result is the existence of a fixed point for the mapping $F$ given by

$$
F\left(w, w_{t}\right) \equiv\left(u, u_{t}\right)
$$

where

$$
u_{t t}-\Delta u+|w|^{k} \partial j\left(u_{t}\right)=|w|^{p-1} u, u=0 \text { on } \partial \Omega, u(0)=u_{0}, u_{t}(0)=u_{1} .
$$

The mapping $F$ is considered on a convex, closed subset of

$$
X_{T} \equiv C\left([0, T] ; H^{1}(\Omega)\right) \cap C^{1}\left([0, T] ; L_{2}(\Omega)\right)
$$

defined by

$$
\begin{aligned}
K_{X_{T}}\left(R, R_{1}\right) \equiv & \left\{w \in X_{T}: w(0)=u_{0} \in H_{0}^{1}(\Omega) \cap H^{2}(\Omega),\right. \text { and } \\
& \left.|w|_{C\left([0, T], H^{1}(\Omega)\right)}+\left|w_{t}\right|_{C\left([0, T] ; H^{1}(\Omega)\right)} \leq R,|w|_{C\left([0, T], W^{2, q}(\Omega)\right)} \leq R_{1}\right\},
\end{aligned}
$$

with the positive constants $R<R_{1}$ and $q=\frac{n}{2}$ is fixed throughout.

Lemma 7.1. Assume $n<5$ and that $k \geq 1,2 \leq p<\frac{4}{n-2}+1, m+1<\frac{p^{*}}{2}$, $k+m<\frac{2 p^{*}}{n}+1$. Then, for any initial data $u_{0} \in H_{0}^{1}(\Omega) \cap H^{2}(\Omega), u_{1} \in H_{0}^{1}(\Omega)$ with

$$
\left|u_{0}\right|_{2, \Omega}+\left|u_{1}\right|_{1, \Omega} \leq R_{0},
$$

there exist positive constants $R\left(R_{0}\right), R_{1}(R)$ and a time $T_{0} \equiv T_{0}\left(R_{1}\right)>0$ such that $F$ has a unique fixed point in $K_{X_{T_{0}}}\left(R, R_{1}\right)$. 
Remark 7.2. The strict inequality $m+1<\frac{p^{*}}{2}$ in the assumptions in Lemma 7.1 is only needed for $n>3$, and it can be replaced by $m+1 \leq \frac{p^{*}}{2}$ in low space dimensions $n \leq 3$. Indeed, for $n \leq 3$, one can work with $W^{2, q}(\Omega)$ instead of $W^{2, \frac{n}{2}}(\Omega)$, where $q=\frac{n}{2}+\sigma$ and $\sigma>0$ is sufficiently small. In this case, the imbedding $W^{2, \frac{n}{2}}(\Omega) \hookrightarrow L_{r}(\Omega), 1 \leq r<\infty$, used in the proof of Lemma 7.1 below will be replaced by $W^{2, q}(\Omega) \hookrightarrow L_{\infty}(\Omega)$.

Proof. We shall first show that $\mathrm{F}$ maps $K_{X_{T}}\left(R, R_{1}\right)$ into itself provided $R\left(R_{0}\right)$, $R_{1}(R)$ are sufficiently large and $T<T_{0}$ where $T_{0}=T_{0}\left(R_{1}\right)$ is sufficiently small. Indeed, we shall prove that $F\left(K_{X_{T_{0}}}\left(R, R_{1}\right) \subseteq K_{X_{T_{0}}}\left(R, R_{1}\right)\right.$ for a sufficiently large $R, R_{1}$ and sufficiently small $T_{0}$. Our the argument here is similar to the argument used in [13. In the proof below, the following Sobolev imbeddings will be used frequently, and sometimes without mention:

$$
H^{1}(\Omega) \hookrightarrow L_{p^{*}}(\Omega) \text { and } W^{2, q}(\Omega) \hookrightarrow L_{r}(\Omega), \text { for } 1 \leq r<\infty .
$$

Step 1. By the same arguments that we used in proving Lemma 3.1, after accounting for additional regularity of $w \in K_{X_{T}}\left(R, R_{1}\right)$ we establish the existence of finite energy solutions $u(t), u_{t}(t)$ which satisfy the following energy inequality:

$$
\begin{aligned}
& |u(t)|_{1, \Omega}^{2}+\left|u_{t}(t)\right|_{0, \Omega}^{2} \leq|u(0)|_{1, \Omega}^{2}+\left|u_{t}(0)\right|_{0, \Omega}^{2} \\
& \quad+C \int_{0}^{t}\left[\left|u_{t}\right|_{0, \Omega}^{2}+\|u\|_{L_{p^{*}}(\Omega)}^{2}\|w\|_{\frac{2 p^{*}(p-1)}{p^{*}-2}}^{2(p-1)}\right] d t \\
& \quad \leq|u(0)|_{1, \Omega}^{2}+\left|u_{t}(0)\right|_{0, \Omega}^{2}+C \int_{0}^{t}\left[\left|u_{t}\right|_{0, \Omega}^{2}+|u|_{1, \Omega}^{2}|w|_{W^{2, q}(\Omega)}^{2(p-1)}\right] d t .
\end{aligned}
$$

Step 2. In order to gain control of the spatial derivative of $u_{t}$ we consider the equation satisfied by $z \equiv u_{t}$. This leads us to study the equation

$$
z_{t t}-\Delta z+|w|^{k} j^{\prime \prime}\left(u_{t}\right) z_{t}=-\left(\frac{d}{d t}|w|^{k}\right) \partial j\left(u_{t}\right)+\frac{d}{d t}\left(|w|^{p-1} u\right),
$$

where (7.3) is understood in the sense of distributions. Since (7.3) is a linear equation in $z$, then all the calculations below can be justified via classical linear arguments (density-based arguments). It should be noted here that if $j$ is not $C^{2}$, then it needs to be approximated by a smooth function. However, this will not affect the final estimates. Indeed, the bounds obtained do not depend on the approximation, and thus, the passage to the limit will recover the original form.

By applying standard energy estimates for equation (7.3) and exploiting the positivity of $j^{\prime \prime}$, we obtain

$$
\begin{aligned}
|z(t)|_{1, \Omega}^{2}+\left|z_{t}(t)\right|_{0, \Omega}^{2} \leq|z(0)|_{1, \Omega}^{2}+\left|z_{t}(0)\right|_{0, \Omega}^{2} & \\
& +C \int_{0}^{t} h(s) d s+\int_{0}^{t}\left|z_{t}(s)\right|_{0, \Omega}^{2} d s
\end{aligned}
$$

where

$h(s)=k^{2} \int_{\Omega}|w|^{2 k-2}\left|w_{t}\right|^{2}|z|^{2 m} d x+\int_{\Omega}\left[(p-1)^{2}|w|^{2(p-2)}\left|w_{t}\right|^{2}|u|^{2}+|w|^{2(p-1)} z^{2}\right] d x$.

We first note that

$$
z(0)=u_{1} \in H_{0}^{1}(\Omega), z_{t}(0)=\Delta u_{0}-|w(0)|^{k} \partial j\left(u_{1}\right)+|w(0)|^{p-1} u(0) .
$$


Since $w(0)=u_{0} \in H^{2}(\Omega) \cap H_{0}^{1}(\Omega)$, we then have

$$
\begin{aligned}
\left\||w(0)|^{k} \partial j\left(u_{1}\right)\right\|_{L_{2}(\Omega)} & \leq C\left(\left\|\left|u_{0}\right|^{k}\left|u_{1}\right|^{m}\right\|_{L_{2}(\Omega)}+\left\|\left|u_{0}\right|\right\|_{L_{2 k}(\Omega)}^{k}\right) \\
& \leq C\left(\left|u_{0}\right|_{2, \Omega}^{k}+\left\|u_{0}\right\|_{L_{\frac{2 k p^{*}}{p^{*}-2 m}}^{k}(\Omega)}^{k}\left\|u_{1}\right\|_{L_{p^{*}}(\Omega)}^{m}\right) \\
& \leq C\left|u_{0}\right|_{2, \Omega}^{k}\left(1+\left|u_{1}\right|_{1, \Omega}^{m} \leq C R_{0}^{k}\left(1+R_{0}^{m}\right),\right.
\end{aligned}
$$

where in (7.6) we have used Hölder's inequality, (7.1), $m \leq \frac{p^{*}}{2}$ (since $m+1<\frac{p^{*}}{2}$ ) and the assumptions on the initial data $u_{0}$ and $u_{1}$. Similarly, we have

$$
\left\||w(0)|^{p-1} u_{0}\right\|_{L_{2}(\Omega)}=\left\|u_{0}\right\|_{L_{2 p}(\Omega)}^{p} \leq C\left|u_{0}\right|_{2, \Omega}^{p} \leq C R_{0}^{p} .
$$

Since $u_{0} \in H^{2}(\Omega) \cap H_{0}^{1}(\Omega)$, it then follows from (7.5) - (7.7) that there exists a positive constant $C\left(R_{0}\right)$ (where $C\left(R_{0}\right)$ depends on $R_{0}$ and the parameters $k, m, p$ ) such that

$$
|z(0)|_{1, \Omega}^{2}+\left|z_{t}(0)\right|_{0, \Omega}^{2} \leq C\left(R_{0}\right) .
$$

In order to estimate the integrals on the right side of (7.4) represented by the function $h(s)$ we apply Hölder's inequality along with the restrictions imposed on the parameters $m, k, p$. We begin with the term containing the nonlinear damping. We first note the assumption $m+1<\frac{p^{*}}{2}$, and so by using Hölder's inequality with the conjugate exponents $\left\{\frac{p^{*}}{P^{*}-2(m+1)}, \frac{p^{*}}{2}, \frac{p^{*}}{2 m}\right\}$, we have

$$
\int_{\Omega}|w|^{2(k-1)}\left|w_{t}\right|^{2}|z|^{2 m} d x \leq\|w\|_{\frac{2(k-1) p^{*}}{P^{*}-2(m+1)}(\Omega)}^{2(k-1)}\left\|w_{t}\right\|_{L_{p^{*}}(\Omega)}^{2}\|z\|_{L_{p^{*}}(\Omega)}^{2 m} .
$$

By the virtue of the Sobolev embeddings in (7.1), we infer

$$
\int_{\Omega}|w|^{2(k-1)}\left|w_{t}\right|^{2}|z|^{2 m} d x \leq C|w|_{W^{2, q}(\Omega)}^{2 k-2}\left|w_{t}\right|_{1, \Omega}^{2}|z|_{1, \Omega}^{2 m} .
$$

Let $0<\epsilon<1 / 2$; then for $n<5$ we have $\frac{2(1-\epsilon) p^{*}}{p^{*}-2-2 \epsilon} \leq p^{*}$. Therefore, we similarly obtain

$$
\begin{aligned}
\int_{\Omega}|w|^{2(p-2)}\left|w_{t}\right|^{2}|u|^{2} d x & =\int_{\Omega}|w|^{2(p-2)}\left|w_{t}\right|^{2}|u|^{2 \epsilon}|u|^{2-2 \epsilon} d x \\
& \leq\left\|w_{t}\right\|_{L_{p^{*}}(\Omega)}^{2}\|w\|_{L_{\underline{2(p-2) p^{*}}}^{2(p-2)}}^{2(\Omega-2)}\|u\|_{L_{\frac{(2-2 \epsilon) p^{*}}{p^{*}-2-2 \epsilon}}^{2-2 \epsilon}}(\Omega) \\
& \leq C\left|w \|_{L_{2 p^{*}}(\Omega)}^{2 \epsilon}\right|_{W^{2, q}(\Omega)}^{(p-2)}\left|w_{t}\right|_{1, \Omega}^{2}|u|_{1, \Omega}^{2-2 \epsilon}|u|_{W^{2, q}(\Omega)}^{2 \epsilon} \\
& \leq C_{\epsilon}|w|_{W^{2, q}(\Omega)}^{2(p-2)}\left|w_{t}\right|_{1, \Omega}^{2}\left(|u|_{1, \Omega}^{2}+|u|_{W^{2, q}(\Omega)}^{2}\right) .
\end{aligned}
$$

We note here that in (7.11) we have an added difficulty due the presence of the term $|u|_{W^{2, q}(\Omega)}^{2}$, which we now estimate. To do this, we will be analyzing the regularity of the elliptic problem:

$$
\Delta u=u_{t t}+|w|^{k} \partial j\left(u_{t}\right)-|w|^{p-1} u \text { in } \Omega, u=0 \text { on } \Gamma .
$$

In what follows we note that $\frac{n}{2}=q \leq 2 \leq p^{*}$ for $n \leq 4$. By recalling the assumption that $p-1<\frac{2 p^{*}}{n}=\frac{4}{n-2}$, we can choose $s \in(0,1)$ such that $\frac{n}{2}(s+p-1)<p^{*}$, 
and set $\rho=\frac{(1-s) q p^{*}}{p^{*}-(s+p-1) q}$. Then, by applying in succession Hölder's and Young's inequalities, we have

$$
\begin{aligned}
\left\||w|^{p-1} u\right\|_{L_{q}(\Omega)} & =\left(\int_{\Omega}|u|^{s q}|u|^{(1-s) q}|w|^{(p-1) q} d x\right)^{\frac{1}{q}} \\
& \leq C\|u\|_{L_{\rho}(\Omega)}^{1-s}\|u\|_{L_{p^{*}}(\Omega)}^{s}\|w\|_{L_{p^{*}}(\Omega)}^{p-1} \\
& \leq C|u|_{W^{2, q}(\Omega)}^{1-s}|u|_{1, \Omega}^{s}|w|_{1, \Omega}^{p-1} \\
& \leq \epsilon|u|_{W^{2, q}(\Omega)}+C_{\epsilon, s}|u|_{1, \Omega}|w|_{1, \Omega}^{\frac{p-1}{s}}
\end{aligned}
$$

where $0<\epsilon<1$, which will be selected shortly. Similarly, by using the assumption $k+m-1<\frac{2 p^{*}}{n}$ we estimate the $L_{q}$ norm of the damping term as follows:

$$
\begin{aligned}
\left\||w|^{k} \partial j\left(u_{t}\right)\right\|_{L_{q}(\Omega)} & \leq C\left(\int_{\Omega}|w|^{q(1-r)}|w|^{(k-1+r) q}\left|u_{t}\right|^{m q} d x\right)^{\frac{1}{q}} \\
& \leq C\|w\|_{L_{\theta}(\Omega)}^{1-r}\|w\|_{L_{p^{*}}(\Omega)}^{k-1+r}\left\|u_{t}\right\|_{L_{p^{*}}(\Omega)}^{m} \\
& \leq C|w|_{W^{2, q}(\Omega)}^{1-r}|w|_{1, \Omega}^{k-1+r}\left|u_{t}\right|_{1, \Omega}^{m},
\end{aligned}
$$

where $\theta=\frac{(1-r) q p^{*}}{p^{*}-(k-1+r+m) q}$ and $0<r<1$ is small enough so that $(k-1+r+m) q<$ $p^{*}$. By using the elliptic regularity for the $W^{2, q}$-spaces applied to (7.12) along with the estimates in (7.13), (7.14), and noting that $\frac{n}{2}=q \leq 2$, we have

$$
\begin{aligned}
|u(t)|_{W^{2, q}(\Omega)} & \leq C\left(\|\left. u_{t t}\right|_{L_{2}(\Omega)}+\epsilon|u|_{W^{2, q}(\Omega)}\right. \\
& \left.+C_{\epsilon, s}|u|_{1, \Omega}|w|_{1, \Omega}^{\frac{p-1}{s}}+|w|_{W^{2, q}(\Omega)}^{1-r}|w|_{1, \Omega}^{k-1+r}\left|u_{t}\right|_{1, \Omega}^{m}\right) .
\end{aligned}
$$

By selecting suitably small $\epsilon$, one has

$$
\begin{aligned}
& |u(t)|_{W^{2, q}(\Omega)} \\
& \leq C\left(\left\|u_{t t}\right\|_{L_{2}(\Omega)}+|u|_{1, \Omega}|w|_{1, \Omega}^{\frac{p-1}{s}}+|w|_{W^{2, q}(\Omega)}^{1-r}|w|_{1, \Omega}^{k-1+r}\left|u_{t}\right|_{1, \Omega}^{m}\right)
\end{aligned}
$$

or

$$
\begin{aligned}
& |u(t)|_{W^{2, q}(\Omega)}^{2} \\
& \quad \leq C\left(\left\|u_{t t}\right\|_{L_{2}(\Omega)}^{2}+|u|_{1, \Omega}^{2}|w|_{1, \Omega}^{\frac{2(p-1)}{s}}+|w|_{W^{2, q}(\Omega)}^{2(1-r)}|w|_{1, \Omega}^{2(k-1+r)}\left|u_{t}\right|_{1, \Omega}^{2 m}\right) .
\end{aligned}
$$

By combining (17.17) and (7.11), and recalling that $w \in K_{X_{T}}\left(R, R_{1}\right)$, we have

$$
\begin{aligned}
\int_{\Omega}|w|^{2(p-2)}\left|w_{t}\right|^{2}|u|^{2} d x & \leq C_{\epsilon}|w|_{W^{2, q}(\Omega)}^{2(p-2)}\left|w_{t}\right|_{1, \Omega}^{2}\left(|u|_{1, \Omega}^{2}+\|\left. u_{t t}\right|_{L_{2}(\Omega)} ^{2}\right. \\
& \left.+|u|_{1, \Omega}^{2}|w|_{1, \Omega}^{\frac{2(p-1)}{s}}+|w|_{W^{2, q}(\Omega)}^{2(1-r)}|w|_{1, \Omega}^{2(k-1+r)}\left|u_{t}\right|_{1, \Omega}^{2 m}\right) \\
\leq C R_{1}^{2(p-1)}\left(|u|_{1, \Omega}^{2}\right. & \left.\left.+\|\left. u_{t t}\right|_{L_{2}(\Omega)} ^{2}+R_{1}^{\frac{2(p-1)}{s}}|u|_{1, \Omega}^{2}+R_{1}^{2 k}\left|u_{t}\right|_{1, \Omega}^{2 m}\right)\right) .
\end{aligned}
$$

We also similarly have

$$
\begin{array}{r}
\int_{\Omega}|w|^{2(p-1)} z^{2} d x \leq C\|z\|_{L_{p^{*}}(\Omega)}^{2}\|w\|_{\frac{2 p^{*}(p-1)}{p^{*}-2}}^{2 p-2}(\Omega) \\
\leq C|w|_{W^{2, q}(\Omega)}^{2(p-1)}|z|_{1, \Omega}^{2} .
\end{array}
$$


By noting that $w \in K_{X_{T}}\left(R, R_{1}\right)$, it then follows from (7.10), (7.11) and (7.19) that $(7.20)$

$$
\begin{aligned}
h(\tau) \leq & \left.C R_{1}^{2(p-1)}\left(|u(\tau)|_{1, \Omega}^{2}+\left\|u_{t t}(\tau)\right\|_{L_{2}(\Omega)}^{2}+R_{1}^{\frac{2(p-1)}{s}}|u(\tau)|_{1, \Omega}^{2}+R_{1}^{2 k}\left|u_{t}(\tau)\right|_{1, \Omega}^{2 m}\right)\right) \\
+ & {\left[R_{1}^{2 k}|z(\tau)|_{1, \Omega}^{2 m}+R_{1}^{2(p-1)}|u(\tau)|_{1, \Omega}^{2}+R_{1}^{2(p-1)}|z(\tau)|_{1, \Omega}^{2}\right], }
\end{aligned}
$$

where $C>0$ is independent of $R_{1}$. Therefore, it follows from (7.4), (7.5) and (17.20) that

$$
\begin{aligned}
|z(t)|_{1, \Omega}^{2}+\left|z_{t}(t)\right|_{0, \Omega}^{2} \leq & C\left(R_{0}\right)+C \int_{0}^{t} R_{1}^{2(p-1)}\left|z_{t}(\tau)\right|_{0, \Omega}^{2} d \tau \\
& +C \int_{0}^{t}\left[R_{1}^{2 k}\left(1+R_{1}^{2(p-1)}\right)|z(\tau)|_{1, \Omega}^{2 m}\right. \\
& \left.+R_{1}^{\frac{2(p-1)(1+s)}{s}}|u(\tau)|_{1, \Omega}^{2}+R_{1}^{2(p-1)}|z(\tau)|_{1, \Omega}^{2}\right] d \tau
\end{aligned}
$$

where $C\left(R_{0}\right)$ is a positive constant depending on $R_{0}$. At this end, we let

$$
\phi(t) \equiv 1+|u(t)|_{1, \Omega}^{2}+|z(t)|_{1, \Omega}^{2}+\left|z_{t}(t)\right|_{0, \Omega}^{2}
$$

Then, it follows from (7.2), (7.4) and (7.21) that

$$
\begin{array}{r}
\phi(t) \leq C\left(R_{0}\right)+C \int_{0}^{t}\left[\left(R_{1}^{2(p-1)}+R_{1}^{\frac{2(p-1)(1+s)}{\tau}}\right) \phi(s)\right. \\
\left.+R_{1}^{2 k}\left(1+R_{1}^{2(p-1)}\right) \phi(\tau)^{m}\right] d \tau .
\end{array}
$$

If $m \leq 1$, then Gronwall's inequality yields

$$
\phi(t) \leq C\left(R_{0}\right) e^{C_{R_{1}} t}, \text { for } 0 \leq t \leq T \leq T_{0},
$$

where $C_{R_{1}}$ is a positive constant that depends on $R_{1}$.

Now, we can select $R=R\left(R_{0}\right)>0$ large enough (we can choose $R^{2}=2 C\left(R_{0}\right)$ say) and then choose $T_{0}\left(R_{1}\right)$ small enough so that $C\left(R_{0}\right) e^{C_{R_{1}} T_{0}} \leq R^{2}$. We note here that we imposed no conditions on $R_{1}$ thus far. Therefore, we have in this case

$$
|u(t)|_{1, \Omega}^{2}+|z(t)|_{1, \Omega}^{2}+\left|z_{t}(t)\right|_{0, \Omega}^{2} \leq R^{2}, \text { for } 0 \leq t \leq T_{0}
$$

For the case $m>1$ we note that (7.22) implies

$$
\phi(t) \leq C\left(R_{0}\right)+C_{R_{1}} \int_{0}^{t} \phi(\tau)^{m} d \tau
$$

for some $C_{R_{1}}>0$. By using a standard comparison theorem, we infer that

$$
\phi(t) \leq\left[C\left(R_{0}\right)^{-(m-1)}-(m-1) C_{R_{1}} t\right]^{\frac{-1}{m-1}},
$$

where the above bound holds for all $t \leq T_{0}$ such that

$$
C\left(R_{0}\right)^{-(m-1)}>(m-1) C_{R_{1}} T_{0} .
$$

We can choose $R=R\left(R_{0}\right)>0$ large enough (say $R^{2}=2 C\left(R_{0}\right)$, for example) and then choose $T_{0}\left(R_{1}\right)$ small enough so that

$$
\left[C\left(R_{0}\right)^{-(m-1)}-(m-1) C_{R_{1}} T_{0}\right]^{\frac{-1}{m-1}} \leq R^{2} .
$$

Hence, in this case one has

$$
|u(t)|_{1, \Omega}^{2}+|z(t)|_{1, \Omega}^{2}+\left|z_{t}(t)\right|_{0, \Omega}^{2} \leq R^{2} \text {, for } 0 \leq t \leq T_{0}\left(R_{1}\right),
$$


or equivalently

$$
|u(t)|_{1, \Omega}^{2}+\left|u_{t}(t)\right|_{1, \Omega}^{2}+\left|u_{t t}(t)\right|_{0, \Omega}^{2} \leq R^{2}, \text { for } 0 \leq t \leq T_{0}\left(R_{1}\right)
$$

Step 3 - $W^{2, q}(\Omega)$ membership. In order to complete the proof of invariance of the set $K_{X_{T_{0}}}\left(R, R_{1}\right)$ under the map $F$ we use the $W^{2, q}(\Omega)$ regularity of the solution $u$ which we have established in (7.16). By recalling the definition of $K_{X_{T_{0}}}\left(R, R_{1}\right)$, and since $\left|u_{t t}(t)\right|_{0, \Omega} \leq R$ for $0 \leq t \leq T_{0}$, then it follows from (7.16) that

$$
|u(t)|_{W^{2, q}(\Omega)} \leq C\left(R+R^{1+\frac{p-1}{s}}+R_{1}^{1-r} R^{k-1+r+m}\right) .
$$

By recalling that $0<r<1$ (and so that $1-r<1$ ) and by using Young's inequality one has the estimate

$$
|u(t)|_{W^{2, q}(\Omega)} \leq \delta R_{1}+C_{\delta, r} R^{\frac{k-1-r+m}{r}}+C R^{1+\frac{p-1}{s}}+C R .
$$

Now, by selecting $\delta<1$, and $R_{1}(R)$ sufficiently large with respect to $R$, we then have the final estimate

$$
|u(t)|_{W^{2, q}(\Omega)} \leq R_{1}, t \leq T_{0}\left(R_{1}\right)
$$

This completes the proof of

$$
F\left(K_{X_{T_{0}}}\left(R, R_{1}\right)\right) \subseteq K_{X_{T_{0}}}\left(R, R_{1}\right)
$$

as long as the parameters $p, m, k$ satisfy

$$
m+1<\frac{p^{*}}{2}, p-1<\frac{2 p^{*}}{n}=\frac{4}{n-2}, k+m-1<\frac{2 p^{*}}{n}=\frac{4}{n-2} .
$$

Step 4 - the contraction property. Here we need to show that for any elements $w, v \in B_{X_{T_{0}}}(R)$ we have that

$$
\left|F\left(w, w_{t}\right)(t)-F\left(v, v_{t}\right)(t)\right|_{H^{1}(\Omega) \times L_{2}(\Omega)} \leq \gamma\left|\left(w, w_{t}\right)-\left(v, v_{t}\right)\right|_{C\left(\left[0, T_{0}\right] ; H^{1}(\Omega) \times L_{2}(\Omega)\right)},
$$

where $\gamma<1$ for $t \leq T_{0}$.

Remark 7.3. We note that the above inequality does not imply continuous dependence of solutions with respect to the initial data in the higher topology. Indeed, the contraction property holds for weaker norm, namely the finite energy norm.

The argument for this step is even simpler than before since we already know that $F\left(w, w_{t}\right), F\left(v, v_{t}\right) \in\left(K_{X_{T_{0}}}\left(R, R_{1}\right)\right)$. We let:

$$
\left(u_{1}, u_{1 t}\right)=F\left(w, w_{t}\right),\left(u_{2}, u_{2 t}\right)=F\left(v, v_{t}\right), U \equiv u_{1}-u_{2} .
$$

Then, with this notation, we have

$U_{t t}-\Delta U+|w|^{k}\left(\partial j\left(u_{1 t}\right)-\partial j\left(u_{2 t}\right)\right)=-\left(|w|^{k}-|v|^{k}\right) \partial j\left(u_{2 t}\right)+|w|^{p-1} u_{1}-|v|^{p-1} u_{2}$.

By applying standard energy estimates and exploiting the monotonicity of $\partial j(s)$ we obtain the estimate

$$
\begin{aligned}
& |U(t)|_{1, \Omega}^{2}+\left|U_{t}(t)\right|_{0, \Omega}^{2} \leq C \int_{0}^{t} \int_{\Omega}\left|U_{t}\right||w-v|\left(|w|^{k-1}+|v|^{k-1}\right)\left|u_{2 t}\right|^{m} d x d s \\
& +C \int_{0}^{t} \int_{\Omega}\left[\left|U_{t}\right||w-v|\left(|w|^{p-2}+|v|^{p-2}\right)\left|u_{1}\right|+\left|U_{t}\right||U||v|^{p-1}\right] d x d s .
\end{aligned}
$$


Thanks to Hölder's and Sobolev's inequalities with the restriction $m+1 \leq \frac{p^{*}}{2}$, we obtain

$$
\begin{aligned}
|U(t)|_{1, \Omega}^{2}+\left|U_{t}(t)\right|_{0, \Omega}^{2} & \\
\leq & C \int_{0}^{t}\left|U_{t}\right|_{0, \Omega}|w-v|_{1, \Omega}\left[| u _ { 2 t } | _ { 1 , \Omega } ^ { m } \left(|w|_{L_{\infty}(\Omega)}^{k-1}\right.\right. \\
& \left.\left.\quad+|v|_{L_{\infty}(\Omega)}^{k-1}\right)+\left|u_{1}\right|_{1, \Omega}\left(|w|_{L_{\infty}(\Omega)}^{p-2}+|v|_{L_{\infty}(\Omega)}^{p-2}\right)\right] d s \\
& \quad+C \int_{0}^{t}\left|U_{t}\right|_{0, \Omega}|U|_{1, \Omega}|v|_{L_{\infty}(\Omega)}^{p-1} d s \\
\leq & 2 C\left(R_{1}^{m+k-1}+R_{1}^{p-1}\right) \int_{0}^{t}\left[\left|U_{t}\right|_{0, \Omega}|w-v|_{1, \Omega}+\left|U_{t}\right|_{0, \Omega}|U|_{1, \Omega}\right] d s .
\end{aligned}
$$

By Young's inequality, we have

$$
\begin{array}{r}
|U(t)|_{1, \Omega}^{2}+\left|U_{t}(t)\right|_{0, \Omega}^{2} \leq C_{R_{1}} \int_{0}^{t}\left[|w-v|_{1, \Omega}^{2}+|U|_{1, \Omega}^{2}+\left|U_{t}\right|_{0, \Omega}^{2}\right] d s \\
\quad \leq C_{R_{1}} T_{1}|w-v|_{C\left(\left[0, T_{1}\right] ; H^{1}(\Omega)\right)}^{2}+C_{R} \int_{0}^{t}\left[|U|_{1, \Omega}^{2}+\left|U_{t}\right|_{0, \Omega}^{2}\right] d s,
\end{array}
$$

for $t \in\left[0, T_{1}\right]$. Hence, Gronwall's inequality yields

$$
|U(t)|_{1, \Omega}^{2}+\left|U_{t}(t)\right|_{0, \Omega}^{2} \leq C_{R} T_{1} \exp \left(C_{R} T_{1}\right)|w-v|_{C\left(\left[0, T_{1}\right] ; H^{1}(\Omega)\right)}^{2},
$$

for $t \in\left[0, T_{1}\right]$. By choosing $0<T_{1} \leq T_{0}$ sufficiently small, then (7.36) yields the desired conclusion in (7.32). The continuity in time of the map $F$ with respect to the $X_{T_{0}}$ topology for elements in $K_{X_{T}}\left(R, R_{1}\right)$ is straightforward. The proof of the lemma is completed.

\section{Proper proof of Theorem 1.10 .}

Proof. By the virtue of Lemma 7.1 and the contraction mapping principle we obtain a unique (local) solution $u$ to the equation

$$
u_{t t}-\Delta u+|u|^{k} \partial j\left(u_{t}\right)=|u|^{p-1} u,
$$

with the smooth initial data $u_{0} \in H^{2}(\Omega), u_{1} \in H^{1}(\Omega)$, where $u$ satisfies

$$
u \in C\left(\left[0, T_{0}\right], H_{0}^{1}(\Omega) \cap L_{\infty}(\Omega)\right), u_{t} \in C\left(\left[0, T_{0}\right], H_{0}^{1}(\Omega)\right), u_{t t} \in C\left(\left[0, T_{0}\right], L_{2}(\Omega)\right) .
$$

In order to complete the proof of Theorem 1.10 it suffices to establish the $H^{2}$ regularity of $u(t)$. This follows by reading off the regularity of $\Delta u$ from the equation

$$
\Delta u=u_{t t}-|u|^{k} \partial j\left(u_{t}\right)+|u|^{p-1} u \in C\left(\left[0, T_{0}\right] ; L_{2}(\Omega)\right), u=0 \text { on } \partial \Omega,
$$

where the regularity of the right-hand side follows from (17.28), the growth condition imposed on $\partial j$ in Assumption 1.1 and the Sobolev embedding theorem. Indeed, since $|u|^{k} \in L_{\infty}(\Omega),\left|u_{t}\right|^{m} \in L_{\frac{p^{*}}{m}}(\Omega)$ and $m \leq \frac{1}{2} p^{*}$, we have

$$
|u|^{k} \partial j\left(u_{t}\right) \in C\left(\left[0, T_{0}\right], L_{2}(\Omega)\right) .
$$

Therefore, the classical elliptic theory implies

$$
u \in C\left(\left[0, T_{0}\right] ; H^{2}(\Omega)\right)
$$

as desired which completes the proof of the first statement in Theorem 1.10,

For the second statement we shall use the fact that the parameters $m, k, p$ are further restricted. In fact, in this case, we can prove global a priori bounds for 
the higher derivatives. This allows to propagate regularity of solutions indefinitely. The argument is as follows.

Since we already know that finite energy solutions are global (recall $p \leq k+m$ ), i.e.,

$$
|u(t)|_{1, \Omega}^{2}+\left|u_{t}(t)\right|_{0, \Omega}^{2} \leq C\left(\left|u_{0}\right|_{1, \Omega},\left|u_{1}\right|_{0, \Omega}\right) \equiv C_{0} .
$$

Therefore, it suffices to obtain the a priori bounds for $\left|u_{t}\right|_{1, \Omega}$ and $|u(t)|_{2, \Omega}$. In order to do so, we consider the equation satisfied by $z \equiv u_{t}$. Namely, we consider the equation

$$
z_{t t}-\Delta z+|u|^{k} j^{\prime \prime}\left(u_{t}\right) z_{t}=\frac{d}{d t}|u|^{p-1} u+\left(\frac{d}{d t}|u|^{k}\right) \partial j\left(u_{t}\right) .
$$

By exploiting the convexity of $j$, the following standard estimate is valid as long as the solution exists:

$$
\begin{aligned}
& |z(t)|_{1, \Omega}^{2}+\left|z_{t}(t)\right|_{0, \Omega}^{2} \leq|z(0)|_{1, \Omega}^{2}+\left|z_{t}(0)\right|_{0, \Omega}^{2} \\
& \quad+C \int_{0}^{t} \int_{\Omega}\left[|u|^{p-1}\left|u_{t}\right|\left|z_{t}\right|+|u|^{k-1}\left|u_{t}\right|^{m+1}\left|z_{t}\right|\right] d x d t .
\end{aligned}
$$

By using Hölder's inequality we obtain

$$
\int_{\Omega}|u|^{p-1}\left|u_{t}\right|\left|z_{t}\right| d x \leq\|u\|_{L_{p^{*}}(\Omega)}^{p-1}\|z\|_{L_{\alpha}(\Omega)}\left|z_{t}\right|_{0, \Omega},
$$

where $\alpha=\frac{2 p^{*}}{p^{*}-2 p+2}$.

Since $p \leq \frac{p^{*}}{2}$ then $\alpha \leq p^{*}$. Thus, by the virtue of the Sobolev embedding theorem we have

$$
\int_{\Omega}|u|^{p-1}\left|u_{t}\right|\left|z_{t}\right| d x \leq|u|_{1, \Omega}^{p-1}|z|_{1, \Omega}\left|z_{t}\right|_{0, \Omega},
$$

which gives the estimate for the first term on the right side of inequality (7). As for the second term on the right side of (7), we argue as follows:

$$
\int_{\Omega}|u|^{k-1}\left|u_{t}\right|^{m+1}\left|z_{t}\right| d x \leq\left(\int_{\Omega}|u|^{2 k-2}\left|u_{t}\right|^{2 m+2} d x\right)^{1 / 2}\left|z_{t}\right|_{0, \Omega}
$$

and

$$
\begin{aligned}
\int_{\Omega}|u|^{2 k-2}\left|u_{t}\right|^{2 m+2} d x & \leq\left(\int_{\Omega}|u|^{\frac{(2 k-2) p^{*}}{2 k-2}}\right)^{\frac{2 k-2}{p^{*}}}\left(\int_{\Omega}\left|u_{t}\right|^{\frac{p^{*}(2 m+2)}{p^{*}-2 k+2}} d x\right)^{\frac{p^{*}-2 k+2}{p^{*}}} \\
& \leq|u|_{1, \Omega}^{2 k-2}\left\|u_{t}\right\|_{L_{\beta}(\Omega)}^{2 m+2}
\end{aligned}
$$

where $\beta=\frac{p^{*}(2 m+2)}{p^{*}-2 k+2}$. Since $k-1<\frac{p^{*}}{2}$ and $m+k<\frac{p^{*}}{2}$ (implied by $\frac{k}{p^{*}}+\frac{m}{2} \leq \frac{1}{2}$ ), we have that $\beta<p^{*}$. By using the interpolation inequality for $L_{p}$ spaces with the interpolating parameters $q=\frac{1}{m+1}, 1-q=\frac{m}{m+1}$, we obtain

$$
\left|u_{t}\right|_{L_{\beta}(\Omega)} \leq\left|u_{t}\right|_{0, \Omega}^{1-q}\left|u_{t}\right|_{L_{r}(\Omega)}^{q},
$$

where $r=\frac{2 \beta q}{2-\beta(1-q)}$. Computing explicitly

$$
r=\frac{2 p^{*}}{p^{*}-2 k-2-m p^{*}} .
$$


Due to the restriction $\frac{k}{p^{*}}+\frac{m}{2} \leq \frac{1}{2}$ we have that $2<r \leq p^{*}$. Thus

$$
\left\|u_{t}\right\|_{L_{\beta}(\Omega)}^{2 m+2} \leq C\left|u_{t}\right|_{0, \Omega}^{2 m}\left\|u_{t}\right\|_{L_{p^{*}}(\Omega)}^{2}
$$

provided $\frac{k}{p^{*}}+\frac{m}{2} \leq \frac{1}{2}$. Hence,

$$
\int_{\Omega}|u|^{k-1}\left|u_{t}\right|^{m+1}\left|z_{t}\right| d x \leq C|z|_{1, \Omega}\left|z_{t}\right|_{0, \Omega}\left|u_{t}\right|_{0, \Omega}^{m}
$$

By combining (7), (7.41) and (7.44), we obtain

$$
\begin{aligned}
|z(t)|_{1, \Omega}^{2}+\left|z_{t}(t)\right|_{0, \Omega}^{2} & \leq|z(0)|_{1, \Omega}^{2}+\left|z_{t}(0)\right|_{0, \Omega}^{2} \\
& +C \int_{0}^{t}\left[|u|_{1, \Omega}^{p-1}+k\left|u_{t}\right|_{0, \Omega}^{m}\right]|z|_{1, \Omega}\left|z_{t}\right|_{0, \Omega} d s
\end{aligned}
$$

Since

$$
|z(0)|_{1, \Omega}^{2}+\left|z_{t}(0)\right|_{0, \Omega}^{2} \leq C\left(\left|u_{0}\right|_{2, \Omega},\left|u_{1}\right|_{1, \Omega}\right) \equiv C_{1}
$$

and

$$
|u|_{1, \Omega}^{p-1}+k\left|u_{t}\right|_{0, \Omega}^{m} \leq C\left(\left|u_{0}\right|_{1, \Omega},\left|u_{1}\right|_{0, \Omega}\right)
$$

then Gronwall's inequality and (7.45) yield

$$
\left|u_{t}(t)\right|_{1, \Omega}+\left|u_{t t}(t)\right|_{0, \Omega} \leq C_{T}\left(\left|u_{0}\right|_{2, \Omega},\left|u_{1}\right|_{1, \Omega}\right),
$$

for all $t \leq T$, where $T$ is arbitrary.

The $H^{2}$ bound for $u(t)$ can be read off from the elliptic equation

$$
\Delta u=u_{t t}+|u|^{k} \partial j\left(u_{t}\right)-|u|^{p-1} u
$$

where

$$
\left.|| u\right|^{k} \partial j\left(\left.\left(u_{t}\right)\right|_{0, \Omega}+\left.\left.|| u\right|^{p}\right|_{0, \Omega} \leq C\left(|u|_{1, \Omega},\left|u_{t}\right|_{1, \Omega}\right) .\right.
$$

Standard elliptic theory and (7.46) yield

$$
\left.|u(t)|_{2, \Omega} \leq\left. C_{T}\left(\mid u_{0}\right)\right|_{2, \Omega},\left|u_{1}\right|_{1, \Omega}\right)
$$

for all $t \in[0, T]$, and $T>0$ is arbitrary. The proof of the global existence in Theorem 1.10 is complete.

\section{ACKNOWLEDGMENT}

The authors express their gratitude to Professor James Serrin. His comments and suggestions led to marked improvement of the manuscript.

\section{REFERENCES}

[1] R.A. Adams, Sobolev Spaces, Academic Press, New York, 1975. MR0450957 (56:9247)

[2] K. Agre and M.A. Rammaha, Global solutions to boundary value problems for a nonlinear wave equation in high space dimensions, Differential \& Integral Equations 14(11) (2001), 1315-1331. MR1859608 (2002h:35190)

[3] V. Barbu, Nonlinear Differential Equations in Banach Spaces, Nordhoff, 1976. MR0390843 $(52: 11666)$

[4] Dang Dinh Ang and A. Pham Ngoc Dinh, Mixed problem for some semi-linear wave equation with a nonhomogeneous condition, Nonlinear Analysis. Theory, Methods \& Applications 12 (1988), 581-592. MR0945666 (89h:35207)

[5] J. Ball, Remarks on blow-up and nonexistence theorems for nonlinear evolution equations, Quart. J. Math. Oxford (2) 28 (1977), 473-486. MR0473484 (57:13150)

[6] V. Georgiev and G. Todorova, Existence of a solution of the wave equation with nonlinear damping and source terms, J. Differential Equations 109 (1994), 295-308. MR1273304 (95b:35141) 
[7] R.T. Glassey, Blow-up theorems for nonlinear wave equations, Math. Z. 132 (1973), 183-203. MR 0340799 (49:5549)

[8] P. Grisvard, Caractérisation de quelques espaces d'interpolation, Arch. Rat. Mech. Anal. 25 (1967), 40-63. MR0213864 (35:4718)

[9] P. Grisvard, Équations différentielles abstraites, Ann. Sci. École Norm. Sup. 2(4) (1969), 311-395. MF0270209 (42:5101)

[10] A. Haraux Nonlinear Evolution Equations-Global Behaviour of Solutions, Springer Verlag, 1981. MR 0610796 (83d:47066)

[11] K. Jörgens, Das Anfangswertproblem im Grossen für eine Klasse nichtlinearer Wellengleichungen, Math. Z. 77 (1961), 295-308. MR0130462 (24:A323)

[12] H. Koch and I. Lasiecka, Hadamard wellposedness of weak solutions in nonlinear dynamic elasticity-full Von Karman systems, Evolution Equations, Semigroups and Functional Analysis, vol 50, Birkhauser, pp. 197-211, 2002. MR1944164 (2003j:35199)

[13] I. Lasiecka and J. Ong, Global solvability and uniform decays of solutions to quasilinear equations with nonlinear boundary dissipation, Communications in PDE's, vol. 24, pp. 20692107, 1999. MR,1720766 (2001c:35154)

[14] I. Lasiecka, J.L. Lions and R. Triggiani, Nonhomogeneous boundary value problem for second order hyperbolic operators, J. Math. Pures et Appl. 65 (1986), 149-192. MR0867669 (88c:35092)

[15] H.A. Levine, Instability and nonexistence of global solutions of nonlinear wave equations of the form $P u_{t t}=A u+\mathcal{F}(u)$, Trans. Amer. Math. Soc. 192 (1974), 1-21. MR0344697 (49:9436)

[16] H.A. Levine and J. Serrin, Global nonexistence theorems for quasilinear evolution equations with dissipation, Arch. Rat. Mech. Anal. 137 (1997), 341-361. MR1463799 (99b:34110)

[17] H.A. Levine, S.R. Park, and J.M. Serrin, Global existence and nonexistence theorems for quasilinear evolution equations of formally parabolic type, J. Differential Equations 142 (1998), 212-229. MR1492883 (99b:35089)

[18] H.A. Levine, S.R. Park, and J.M. Serrin, Global existence and global nonexistence of solutions of the Cauchy problem for a nonlinearly damped wave equation J. Math. Anal. Appl. 228 (1998), 181-205. MR 1659905 (99k:35124)

[19] J.L. Lions and E. Magenes, Non-Homogeneous Boundary Value Problems and Applications I, II, Springer-Verlag, New York-Heidelberg-Berlin, 1972. MR0350177(50:2670) MR0350178 $(50: 2671)$

[20] J.L. Lions and W.A. Strauss, Some non-linear evolution equations, Bull. Soc. Math. France 93 (1965), 43-96. MR0199519 (33:7663)

[21] L.E. Payne and D. Sattinger, Saddle points and instability of nonlinear hyperbolic equations, Israel Math. J. 22 (1981), 273-303. MR0402291 (53:6112)

[22] D.R. Pitts and M.A. Rammaha, Global existence and non-existence theorems for nonlinear wave equations, Indiana University Math. Journal 51(6) (2002), 1479-1509. MR1948457 (2003j:35219)

[23] P. Pucci and J. Serrin, Global nonexistence for abstract evolution equations with positive initial energy, J. Differential Equations 150 (1998), 203-214. MF 1660250 (2000a:34119)

[24] M.A. Rammaha and T.A. Strei, Global existence and nonexistence for nonlinear wave equations with damping and source terms, Trans. Amer. Math. Soc. 354(9) (2002), 3621-3637. MR 1911514 (2003f:35214)

[25] R. Seeley, Interpolation in $L^{P}$ with boundary conditions, Stud. Math. XLIV (1972), 47-60. MR $0315432(47: 3981)$

[26] I. E. Segal, Non-linear semigroups, Annals of Math. 78 (1963), 339-364. MR0152908 $(27: 2879)$

[27] J. Serrin, G. Todorova, E. Vitillaro, Existence for a nonlinear wave equation with damping and source terms, Differential Integral Equations 16(1) (2003), 13-50. MR1948871 (2003j:35221)

[28] J. Simon, Compact sets in the space $L_{p}(0, T, B)$. Annali Mat. Pura et Applic. 148 (1987), 65-96. MR0916688 (89c:46055)

[29] R. Temam, Navier-Stokes Equations, Theory and Numerical Analysis, North-Holland, 1984. MR 0769654 (86m:76003) 
[30] G. Todorova, Cauchy problem for nonlinear wave equations with nonlinear damping and source terms, C. R. Acad. Sci. Paris Sér. I Math. 326 (1998), 191-196. MR1646932 (99e:35154)

[31] G. Todorova, Cauchy problem for a nonlinear wave equation with nonlinear damping and source terms, Nonlinear Anal. 41 (2000), 891-905. MR1764026|(2001c:35160)

[32] H. Tsutsumi, On solutions of semilinear differential equations in a Hilbert space, Math. Japonicea 17 (1972), 173-193. MR0355247 (50:7723)

[33] G.F. Webb, Existence and asymptotic behavior for a strongly damped nonlinear wave equation, Can. J. Math. 32 (1980), 631-643. MR0586981 (81i:35116)

[34] E. Zeidler, Nonlinear Functional Analysis and its Applications. Springer Verlag, 1986. MR:0816732 (87f:47083)

Department of Mathematics, University "Al. J. Cuza", 6600 Iasi, Romania

E-mail address: vb41@uaic.ro

Department of Mathematics, University of Virginia, Charlottesville, Virginia 229044137

E-mail address: il2v@virginia.edu

Department of Mathematics, University of Nebraska-Lincoln, Lincoln, Nebraska $68588-0130$

E-mail address: rammaha@math.unl.edu 